\title{
Comparison of the Hi-C, GAM and SPRITE methods by use of polymer models of chromatin
}

\author{
Luca Fiorillo1,\#, Francesco Musella1,\#, Rieke Kempfer ${ }^{2,3}$, Andrea M. Chiariello ${ }^{1}$ Simona Bianco ${ }^{1}$, \\ Alexander Kukalev ${ }^{2}$, Ibai Irastorza-Azcarate ${ }^{2}$, Andrea Esposito ${ }^{1}$, Mattia Conte ${ }^{1}$, Antonella Prisco ${ }^{4}$, Ana \\ Pombo ${ }^{2,3,5}$, Mario Nicodemi ${ }^{1,2,5, *}$ \\ ${ }^{1}$ Dipartimento di Fisica, Università di Napoli Federico II, and INFN Napoli, Complesso Universitario di Monte Sant'Angelo, \\ 80126 Naples, Italy. \\ ${ }^{2}$ Berlin Institute for Medical Systems Biology, Max-Delbrück Centre (MDC) for Molecular Medicine, Berlin, Germany. \\ ${ }^{3}$ Humboldt-Universität zu Berlin, 10117 Berlin, Germany \\ ${ }^{4}$ CNR-IGB, via Pietro Castellino 111, Naples, Italy \\ ${ }^{5}$ Berlin Institute of Health (BIH), Berlin, Germany. \\ \# These authors contributed equally \\ * Lead contact: mario.nicodemi@na.infn.it
}

\begin{abstract}
Powerful technologies have been developed to probe chromatin 3D physical interactions genome-wide, such as $\mathrm{Hi}-\mathrm{C}, \mathrm{GAM}$ and SPRITE. Due to their intrinsic differences and without a benchmarking reference, it is currently difficult to assess how well each method represents the genome 3D structure and their relative performance. Here, we develop a computational approach to implement Hi-C, GAM and SPRITE in-silico to compare the three methods in a simplified, yet controlled framework against known polymer 3D structures. We test our approach on models of three 6-Mb genomic regions, around the Sox9 and the HoxD genes in mouse ES cells, and around the Epha4 gene in mouse CHLX-12 cells. The modelderived contact matrices consistently match $\mathrm{Hi}-\mathrm{C}, \mathrm{GAM}$ and SPRITE experiments. We show that in-silico $\mathrm{Hi}-\mathrm{C}, \mathrm{GAM}$ and SPRITE average data are overall faithful to the 3D structures of the polymer models. We find that the inherent variability of model single-molecule 3D conformations and experimental efficiency differently affect the contact data of the different methods. Similarly, the noise-to-signal levels vary with genomic distance differently in in-silico Hi-C, SPRITE and GAM. We benchmark the performance of each technology in bulk and in single-cell experiments, and identify the minimal number of cells required for replicates to return statistically consistent chromatin contact measures. Under the same experimental conditions, SPRITE requires the lowest number of cells, Hi-C is close to SPRITE, while GAM is the most reproducible method to capture interactions at large genomic distances.
\end{abstract}




\section{INTRODUCTION}

The 3-dimensional (3D) organization of chromosomes in the nucleus of cells has a crucial role in the regulation of genomic activities and transcriptional programs ${ }^{1-7}$. To access genome-wide information on chromatin architecture and DNA interactions, a number of sequencing approaches are currently being developed ${ }^{8}$, while high-resolution microscopy is rapidly advancing ${ }^{9-12}$. Sequencing approaches include 3C-based methods, such as $\mathrm{Hi}-\mathrm{C}$ and its developments ${ }^{13-19}$, Genome Architecture Mapping $(G A M)^{20}$ and Split-Pool Recognition of Interactions by Tag Extension (SPRITE) ${ }^{21}$. These approaches have shown that the mammalian genome has a complex 3D organization where functional contacts occur across distal DNA regions, such as loops between enhancers and promoters ${ }^{15}$, along with interactions at the megabase scale within Topologically Associated Domains (TADs) ${ }^{22,23}$ and higher-order structures such as metaTADs ${ }^{24}$ and $A / B$ compartments ${ }^{13}$. However, it remains unclear to what extent these technologies are faithful to the underlying 3D structure of the genome and whether they measure different aspects of chromosomal 3D organization. Since they return distinct measures of chromatin interactions, it is also difficult to identify a clear benchmark to compare their performances in different conditions.

$\mathrm{Hi}-\mathrm{C}$ methods have revolutionised the field of chromosome architecture and are widely used. They provide a measure of the abundance of pairwise interactions, i.e., a Hi-C contact frequency map, by sequencing the ligation products of DNA fragments that are in close spatial proximity in the nucleus ${ }^{13,15}$. GAM probes 3D proximity of DNA sites by sequencing the genomic content of thin cryo-sectioned and laser micro-dissected slices from the nuclei of cells fixed in optimal preservation conditions ${ }^{8,20}$. Physically distant DNA sites are unlikely to co-segregate in the same thin slice, whereas physically proximal sites tend to co-segregate. The output of a GAM experiment is a segregation table indicating which loci are present in each slice, based on sequencing its DNA content. To identify pair-wise or multiways contacts, a GAM co-segregation map is calculated, i.e., the frequency with which pairs (or groups) of genomic regions are found in the same slices. From GAM data single-cell DNA non-random interaction probabilities can be reconstructed by use of statistical tools, such as SLICE ${ }^{20}$. Finally, SPRITE ${ }^{21}$ relies on the sequencing of barcoded DNA: after DNA crosslinking and fragmentation in isolated nuclei (as in $\mathrm{Hi}-\mathrm{C}$ ), interacting chromatin complexes are uniquely barcoded via a split-pool method and identified by sequencing. SPRITE interaction maps can be extracted from analysing the DNA segments that have the same barcode, which must originate from the same interacting complex.

To compare Hi-C, GAM and SPRITE, we run a computational experiment that implements the three methods in-silico on an ensemble of known 3D polymer structures and analyse their outputs in a simplified, yet fully controlled framework. To facilitate the comparison with real experimental data, rather than using arbitrary polymer conformations, we focused on the polymer models of two genomic regions around the Sox 9 and the HoxD genes from mouse embryonic stem cells (mESC), respectively $6 \mathrm{Mb}$ and $7 \mathrm{Mb}$ long ${ }^{25,26}$. We also considered the model of a $6 \mathrm{Mb}$ long region around the Epha4 gene from mouse $\mathrm{CHLX}-12$ cells $^{27}$. The comparison of the performance of the different technologies in those loci is interesting also because, for instance, disease-linked structural variants located around the Sox 9 and Epha4 genes have been shown to induce gene mis-expression as a consequence of the rewiring of contacts with local enhancers ${ }^{6,27,28}$; and the HoxD locus presents a complex 3D compartmentalization which is thought to have a broad functional role in controlling transcriptional states during differentiation ${ }^{29,30}$. Different computational approaches ${ }^{31-34}$ and polymer models ${ }^{25,27,35-44}$ have been 
discussed to reconstruct chromatin 3D conformations. Here, we focused on the String\&Binders (SBS) polymer model ${ }^{27,37,39}$ because it has been already validated against $\mathrm{Hi}-\mathrm{C}$ data in those loci ${ }^{25-27}$. The polymer models of the considered loci were inferred from Hi-C data and used to derive an ensemble of known 3D structures for each locus. Those 3D structures were in turn employed to benchmark the performances of Hi-C, SPRITE and GAM in bulk as well as in single-cell computational experiments. For the Sox9 locus, a polymer model inferred from GAM data has been also analysed ${ }^{45}$ and returned similar results. Finally, as a control, we also ran investigations in a toy block-copolymer model, unrelated to any chromosomal region, which returned a similar scenario about the performance of the three technologies.

Here, we show that in-silico average $\mathrm{Hi}-\mathrm{C}, \mathrm{GAM}$ and SPRITE matrices match their corresponding experimental bulk data validating our approach and the polymer models chosen. We found that bulk in-silico Hi-C, GAM and SPRITE data are all faithful to the reference 3D architecture as they have high correlations with the benchmark average distance matrix of our polymer structures. In contrast, singlecell data are affected by high levels of variability even in the case of ideal detection efficiency because of the inherent variety of single-molecule conformations of the polymer ensembles. Finally, we show that detection efficiency and number of single-molecule structures (a proxy for single cells) considered in the in-silico experiments differently affect contact data and the noise-to-signal ratio at different genomic distances across the three technologies. While poor efficiencies can be compensated by large cell numbers, we found that GAM is significantly less noise affected at larger genomic distances than the other methods, under similar experimental conditions (e.g., a given detection efficiency), but requires a comparatively much larger number of cells to ensure similarity across replicates, SPRITE requiring the least and $\mathrm{Hi}-\mathrm{C}$ close to SPRITE.

\section{RESULTS}

\section{Derivation of in-silico Hi-C, SPRITE, and GAM interaction maps from known single-molecule 3D structures}

To compare in-silico Hi-C, GAM and SPRITE data, we focused first on the case study of a 6Mb-wide region around the Sox9 gene (chr11:109Mb-115Mb, mm9) in mESCs. The SBS polymer model of that locus had been previously developed and shown to well reproduce $\mathrm{Hi}-\mathrm{C}$ data ${ }^{25}$. The SBS is a model of chromatin describing the textbook picture where molecules, such as transcription factors, form DNA loops by bridging distal cognate binding sites $^{37}$ (see also Materials and Methods). The SBS model has been shown to well describe Hi-C, GAM and FISH data across loci and cell types ${ }^{20,24-27,39,45-47}$. The genomic locations of the binding sites of the model of the Sox9 locus were inferred from its Hi-C data ${ }^{23}$ by the PRISMR algorithm ${ }^{25,27}$, which finds the minimal set of binding sites (and cognate binders) best describing the input data from only polymer physics (Materials and Methods). Here, we considered the published model of the locus at $40 \mathrm{~kb}$ resolution, with no additional refinements or improvements, and explored an ensemble of single-molecule 3D polymer structures derived by Molecular Dynamics simulations in the thermodynamics steady state of the system (Figure 1a, Materials and Methods).

Next, we computationally implemented the steps of the Hi-C, GAM and SPRITE methods on such ensemble of 3D structures to derive an in-silico proxy of their contact data (Figure 1a). In brief, in insilico $\mathrm{Hi}-\mathrm{C}$, we fragmented in equal segments the two polymer chains representing the two Sox 9 alleles in each cell, ligated cross-linked fragments and counted ligation products to derive an in-silico analogue 
of $\mathrm{Hi}-\mathrm{C}$ contact frequencies (Materials and Methods). The overall efficiency of the process is considered to be the product of the in-silico cross-linking, digestion, biotinylation, ligation and sequencing efficiencies. In-silico SPRITE was analogously implemented, in particular, by counting chain fragments tagged with the same barcode. Finally, in-silico GAM was performed by producing randomly oriented slices out of a sphere (representing the nucleus) where two single-molecule 3D structures (the two "alleles") have been randomly positioned and by listing the polymer segments falling within each slice to derive the co-segregation matrix (see Materials and Methods). The overall efficiency here is the detection and sequencing efficiency of such segments, while the nuclear radius and the slice thickness are parameters set to match typical experimental values ${ }^{20}$ (Materials and Methods).

Such a procedure returns in-silico contact maps from the known 3D structures of the SBS model, providing a simplified, yet fully known benchmark to compare Hi-C, SPRITE and GAM in different contexts. In particular, we investigated how the overall efficiency and the number of pairs, N, of 3D single-molecule structures included in the analysis (below, $\mathrm{N}$ is named, for simplicity, the number of insilico cells) affect the output of the three technologies.

\section{In-silico Hi-C, SPRITE and GAM reproduce experimental data}

As our polymer model is inferred from $\mathrm{Hi}-\mathrm{C}$ data ${ }^{23}$, we checked that the derived in-silico bulk $\mathrm{Hi}-\mathrm{C}$ map, i.e., contact data averaged over the ensemble of 3D structures, reproduces bulk Hi-C experimental interaction frequencies in the Sox 9 locus in $\mathrm{mESC}^{23}$ (Figure 1b). We measured the correlation between the simulated and real $\mathrm{Hi}-\mathrm{C}$ data and found that the Spearman $\left(r_{s}\right)$, Pearson $(r)$ and HiCRep (scc) ${ }^{48}$ coefficients have all high values, respectively $r_{s}=0.83, r=0.83$ and $s c c=0.80$ (Supplementary Table 1a), as previously reported ${ }^{25}$. Analogous results were obtained in the comparison between in-silico and experimental Hi-C maps using the HoxD locus in mESC and the Epha4 locus in CHLX-12 cells (Supplementary Figure 1, Supplementary Figure 3a and Materials and Methods).

Importantly, the in-silico SPRITE and GAM contact matrices derived from the same model 3D structures have also high correlations with the independent SPRITE and GAM experimental data (respectively $r_{s}=0.92$ and $r_{s}=0.79, r=0.75$ and $r=0.80$, and $s c c=0.57$ and $s c c=0.40$, Figure 1b, Supplementary Table 1a). In the comparison, we used published SPRITE bulk mESC data ${ }^{21}$ and a GAM dataset produced for the 4D Nucleome consortium ${ }^{49}$ (Materials and Methods) composed of 1122 nuclear profiles (slices) from F123 mESC cells, here compared with the output from precisely 1122 in-silico slices. Similar results are found for the mESC HoxD locus (Supplementary Figure 1). In particular, the visual differences and lower correlations between experimental and in-silico GAM contact matrices derived from Hi-C-based polymers may also raise the possibility that $\mathrm{Hi}-\mathrm{C}$ and GAM experimental data may capture some different specific features of chromatin contacts, although they could just derive from experimental noise.

Overall, the agreement between model and experiments across independent datasets provides a validation of our polymer model, as the 3D structures inferred from $\mathrm{Hi}-\mathrm{C}$ data only well reproduce independent GAM and SPRITE data too. It also shows that our in-silico approach has no major biases favouring Hi-C, SPRITE or GAM. That supports the view that our ensemble of 3D structures provides a good description of single-cell conformations of the loci and that the approach presented here can work as a simplified, yet useful reference system to compare the performance of the three technologies. 


\section{Bulk Hi-C, SPRITE and GAM data all faithfully describe benchmark average distance matrices}

Next, we investigated how well in-silico Hi-C, SPRITE and GAM data on the Sox9 locus reflect the underlying spatial conformations of the polymers in the ensemble. Towards this aim, we computed the average distance matrix of the known 3D structures and compared it with in-silico Hi-C, SPRITE and GAM bulk data, i.e., the average over a large number of in-silico cells (Figure 2). We found that the three methods have high absolute Spearman correlation coefficients with the average distance matrix $\left(r_{s}<-0.89\right.$, values are negative because large physical distances correspond to small contact frequencies), GAM having the highest, followed by SPRITE and Hi-C (Pearson and HiCRep correlations give analogous results, Supplementary Table $\mathbf{1 b})$.

Interestingly, the patterns visible in the in-silico Hi-C, SPRITE and GAM bulk data are similar to each other, albeit GAM better highlights longer-range contacts between TADs (Figure 2a). In particular, the three in-silico methods all identify TADs that correspond to those previously found by experimental Hi$C^{23}$ (Figure 2b, different colours in the bottom bar). This is consistent with previous genome-wide analyses showing that the location of TAD boundaries in $\mathrm{MESC}$ is highly correlated in Hi-C, SPRITE and $\mathrm{GAM}^{20,21}$. Additionally, the contact patterns in $\mathrm{Hi}-\mathrm{C}$, SPRITE and GAM all reflect the underlying domainlike patterns of the average 3D distance matrix in the model locus, representing the known conformations of the ensemble of single-molecules inferred from Hi-C (Figure $\mathbf{2 b}$ ). Again, analogous results are found for the HoxD and Epha4 loci (Supplementary Figures 2a,b and Supplementary Figures $3 b, c)$.

Taken together, our results support the view that bulk data from $\mathrm{Hi}-\mathrm{C}$, SPRITE and GAM are faithful to the overall spatial structure of the underlying 3D conformations, providing comparable information on the average distance map of the considered Sox9, HoxD and Epha4 locus models.

\section{Stochasticity of single-cell data reflects intrinsic variability of single-molecule 3D conformations}

Whereas bulk interaction matrices are comparatively similar across replicate experiments, single-cell $\mathrm{Hi}-\mathrm{C}$ data exhibit a strong variability ${ }^{50-54}$. Here, we explore how single-cell variability reflects limited detection efficiency and, importantly, the inherent differences across single-molecule conformations of chromatin, whereby even single-cell experiments with $100 \%$ efficiency can return different contact maps (Figure 3).

We discuss first the ideal case of in-silico experiments where the efficiency is set to 1.0. Single-molecule conformations vary widely across the ensemble of 3D structures (Figure 3a, bottom) and their corresponding in-silico single-cell distance matrices (Figure 3a, top) have broadly varying Spearman correlations with the average distance matrix, with a mean of $r_{s}=0.88$ (Figure $3 b$, the values of the mean Pearson and HiCRep correlations are analogous, Supplementary Table 1c). Hence, there are broad structural differences between pairs of single molecules, as manifested in their corresponding singlecell measures. We found that the correlation of an in-silico single-cell Hi-C, SPRITE or GAM contact map (Figure 3c) with its corresponding single-cell distance matrix is much lower than in the case of bulk data discussed before: even in the case with efficiency 1, the average Spearman correlation is around $r_{s}=-$ 0.37 and $r_{s}=-0.46$ for respectively in-silico $\mathrm{Hi}-\mathrm{C}$ and SPRITE (Figure 3d, and Supplementary Table 1d). For GAM the correlation is even lower (average $r_{s}=-0.15$ ) and its distribution much broader, in the range $-0.4<r_{s}<0$. That is also a consequence of the different experimental procedure, because a single-cell 
in-silico Hi-C and SPRITE experiment returns the contacts measured over an entire in-silico nucleus, i.e., two independent polymer structures representing the alleles, whereas a single-cell in-silico GAM experiment probes the polymer content of only a single slice of an in-silico nucleus, i.e., a tiny fraction of the two polymers.

Summarizing, even in the ideal case of a $100 \%$ efficiency experiment, single-cell contact data are drastically less faithful to single-cell distance patterns than bulk data. In particular, single-cell in-silico $\mathrm{Hi}-\mathrm{C}$ and SPRITE maps of the considered $6 \mathrm{Mb}$ locus have a correlation around $\mathrm{r}_{\mathrm{s}}=-0.4$ with their corresponding single-cell distance matrices, whereas GAM has lower and much broader correlations because the experimental procedure samples a single slice rather than a single nucleus.

Contact data from single-cell experiments become further deteriorated, as expected, for lower values of the detection efficiency, and have worse correlations with the corresponding single-cell distance maps (Supplementary Table 1e). Consequently, the variability of replicates from in-silico single-cell experiments increases, i.e., the correlation between their contact maps decreases. For example, for an efficiency of 0.5 , we found that the average correlation between in-silico single-cell replicates is around $r_{s}=0.2,0.4$ and 0.1 for respectively Hi-C, SPRITE and GAM maps. Importantly, the values of correlation found are consistent with those reported in real experimental studies: for example, the average Spearman correlation between different Hi-C maps of the Sox9 locus from real single-cell experiments in CD4 $T_{H} 1$ cells with efficiency approximately around $0.025^{50}$ is $r_{s}=0.01$, which is numerically equal to the Spearman correlation found between in-silico Hi-C maps at the same efficiency in our model of mESC (Materials and Methods and Supplementary Figure 4a). The impact of a limited efficiency on interactions maps is systematically investigated in the next section.

In brief, our results show that in-silico single-cell experiments are inherently broadly varying because they sample different single-molecule 3D structures and, additionally, their contact maps are less faithful to the corresponding single-cell distances. A limited detection efficiency further increases the fluctuations in contact maps to the point that replicates can have correlations well below 0.1 for realistic efficiencies. That appears to be reflected in, and consistent with, the stochastic nature of interactions between DNA sites observed in real single-cell experiments ${ }^{20,50-53}$.

\section{Threshold cell number required for replicate reproducibility differs in Hi-C, SPRITE and GAM}

The quality of in-silico Hi-C, SPRITE and GAM contact maps improves when the number of in-silico cells, $\mathrm{N}$, is increased in the computational experiments (Figure 4). Due to the intrinsic variability of singlemolecule structures, the improvement with $\mathrm{N}$ occurs also in the ideal case of a $100 \%$ efficiency (Figure 4a). Figure $4 \mathbf{b}$ shows, for example, the effect of different $\mathrm{N}$ on the contact matrices of in-silico Hi-C, SPRITE and GAM, in the case where the analyses are run with efficiencies comparable to typical experimental values. We set the $\mathrm{Hi}-\mathrm{C}$ efficiency to 0.05 , taken as an upper limit of values reported in recent studies $50,51,53,55$; the same value is used as an estimate of the efficiency for SPRITE (M. Guttman, personal communication). Since the experimental efficiency in GAM is roughly one order of magnitude larger than $\mathrm{Hi}-\mathrm{C}$ and SPRITE ${ }^{20}$, in the shown example we used an in-silico GAM efficiency of 0.5, a value which is close to the 0.6 efficiency estimated for the mESC dataset used in this work (Materials and Methods).

The patterns of the contact matrices become sharper and stabilise when $\mathrm{N}$ becomes large enough, as 
also observed in experimental investigations ${ }^{50,56}$. Importantly, overall the large $\mathrm{N}$ matrices do not depend on the considered efficiency value and the average over a large number of cells compensates, in general, for reduced efficiencies (see Figure $\mathbf{4 a}$ and $\mathbf{4 b}$ and Materials and Methods). However, our data show that the threshold value of $\mathrm{N}$ to reach saturation in the data strongly depends on the efficiency level and is different in different technologies, as we now illustrate.

We aimed to identify the minimal number of cells that, at a given efficiency, is required for replicate experiments to return similar outputs, i.e., to approach the bulk limit, in the in-silico implementation of $\mathrm{Hi}-\mathrm{C}$, SPRITE and GAM. To measure the similarity of pairs of identical experiments in the Sox 9 locus (each having a given $\mathrm{N}$ and efficiency, Figure 4c), we computed the average Pearson correlation between contact maps (Figures $\mathbf{4 d , e}$; the use of Spearman or HiCRep correlations returned analogous results Supplementary Figure 5). The correlation grows when $N$ is increased and it plateaus to 1 in the large $\mathrm{N}$ limit (Figures $\mathbf{4 d , e}$ ), independently of the efficiency of the in-silico experiment. For each given efficiency, we heuristically define the minimal number of cells, $M$, required for having statistically reproducible results across replicates, as the value of $\mathrm{N}$ where the correlation grows larger than a given threshold, $r_{t}=0.9$ (Materials and Methods). Importantly, we found that $M$ is significantly different in the different technologies: for example, if the efficiency is 0.1 , we found that $M$ is 200,100 and 2000 for respectively $\mathrm{Hi}-\mathrm{C}$, SPRITE and GAM (Figure 4d). Figure 4e shows the correlation between replicates at varying $\mathrm{N}$ obtained for efficiencies close to those reported in real $\mathrm{Hi}-\mathrm{C}, \mathrm{SPRITE}$ and GAM experiments, i.e., as specified above, 0.05 for $\mathrm{Hi}-\mathrm{C}$ and SPRITE and 0.5 for GAM: in those cases, $\mathrm{M}$ is approximately 650, 250 and 800 respectively. Similar behaviours were also found for the HoxD and Epha4 loci (Supplementary Figures $\mathbf{2 c}$ and $\mathbf{3 d}$ ). In general, for a given efficiency, we find that in-silico SPRITE plateaus earlier than $\mathrm{Hi}-\mathrm{C}$, while GAM typically requires a number of cells one order of magnitude larger. However, GAM experiments have currently efficiencies one order of magnitude higher than $\mathrm{Hi}-\mathrm{C}$ or SPRITE, hence the number of cells required for saturation become similar in the three methods.

Next, we checked how the in-silico estimates of $\mathrm{M}$ compares against available systematic experimental investigations. First, we verified that the correlation values between in-silico replicates are comparable to those found in experiments. We considered the 60 different single-cell Hi-C maps produced in CD4 $\mathrm{T}_{\mathrm{H}} 1$ cells ${ }^{50}$ with efficiency around 0.025 and compared their average map with the corresponding bulk $\mathrm{Hi}-\mathrm{C}$ data for the Sox9 locus: the correlation is $r_{s}=0.33$, which is not far from $r_{s}=0.27$ found between insilico $\mathrm{Hi}-\mathrm{C}$ maps in the analogous conditions in mESC (Supplementary Figure $\mathbf{4 b}$ and Materials and Methods). Second, to verify that our estimates of $M$ are consistent with available experimental results we considered the data from a recent Low-C experiment on $\mathrm{mESC}^{56}$ where, in the case of a $10 \mathrm{Mb}$ wide locus, a sample of 1000 cells was shown to be large enough to produce contact maps highly similar to the bulk one (Pearson correlation $r=0.95$ ). That estimate of the minimal number of cells needed to approach the bulk limit is consistent with the above reported value of $\mathrm{M}=650$ for in-silico $\mathrm{Hi}-\mathrm{C}$ for an efficiency equal to 0.05 . Taken together, these examples show that the in-silico estimates of $M$ are informative of real experiments.

Finally, we systematically investigated how the quality of the in-silico data is affected by the efficiency of the experiment (Supplementary Figure 6). In particular, the number of cells required for saturation, $M$, strongly depends on the efficiency (Figure $\mathbf{4 f}$ ): $M$ diverges approximately as an inverse squared power law as the efficiency becomes small. In other words, halving the efficiency requires to quadruple the cell number to achieve the same quality levels. In general, we find that M for SPRITE is two times 
smaller than the corresponding value for $\mathrm{Hi}-\mathrm{C}$ and one order of magnitude smaller than GAM. Additionally, our investigation shows that even in the ideal case of an efficiency equal to 1 , single-cell replicates have below threshold correlations, as $M$ is larger than 10 even for SPRITE due to the intrinsic variability of single-molecule 3D structures, as reported above. That rationalises of the broad variability observed in single-cell $\mathrm{Hi}-\mathrm{C}$ experiments.

We stress that the above definition of $M$ is heuristic, albeit easy to visualise. It is, however, fully consistent with a definition grounded on the Central Limit Theorem (CLT). Consider the average value, $\mu$, and the standard deviation, $\sigma$, of a given entry of a contact map in an experiment with $\mathrm{N}$ cells at a given efficiency. CLT imposes that the noise-to-signal squared ratio, $\sigma^{2} / \mu^{2}$ scales as $1 / \mathrm{N}$. Accordingly, from the $C L T$, the minimal number of cells, $L$, required to make the noise-to-signal squared ratio smaller than a given threshold, $\delta$, is $L=A \delta^{-2} \sigma^{2} / \mu^{2}$, where $A$ is a constant (Materials and Methods). We checked that $M$ and the average value of $L$ are linearly proportional to each other, i.e., $M$ is inversely proportional to the squared signal-to-noise ratio averaged over all the entries of a single-cell contact map, $\rho$ (Materials and Methods and Supplementary Figure 7). Hence, albeit heuristic, the above intuitive definition of $\mathrm{M}$ is grounded on the CLT. With analogous statistical arguments (Materials and Methods) the approximate inverse squared power law relation between $\mathrm{M}$ and the efficiency (Figure 4f) can be explained.

The sets of in-silico single-molecule 3D structures employed in all our analyses were produced using polymer models inferred from $\mathrm{Hi}-\mathrm{C}$ data ${ }^{25-27}$. However, for the Sox9 locus we tested that our results remain overall unchanged also when the polymer 3D structures are constructed from the SBS polymer model of the locus inferred from GAM data rather than $\mathrm{Hi}_{-} \mathrm{C}^{45}$ (Supplementary Figure 8 and Materials and Methods). Additionally, to assess the general validity of our analyses, we applied the in-silico approach to 3D conformations of a toy block-copolymer, unrelated to real chromatin loci, finding similar results (Supplementary Figure 9 and Materials and Methods).

\section{SLICE single-cell interaction probability maps}

Next, we investigated the performance of the GAM data analysis tool SLICE (Supplementary Figure 10). SLICE is a statistical method to identify non-random co-segregation events (i.e., specific interactions) from GAM co-segregation data. In particular, the output of SLICE is the single-cell interaction probability (Pi) of pairs, and multiplets, of DNA sites ${ }^{20}$. Importantly, we found that SLICE bulk interaction probabilities are faithful to the known average distance matrix $\left(r=-0.95, r_{s}=-1.00, s c c=-0.99\right.$, Supplementary Figures $\mathbf{1 0 a , b}$ ). The SLICE matrices behave with $\mathrm{N}$ and with the efficiency as found for GAM contact maps (Figure 4). However, as by definition SLICE specifically detects significant interactions, we found that the average number of in-silico cells, $M$, needed to return statistically reproducible results across replicates is approximately half than the one required for GAM in the same conditions (Supplementary Figures 10c-e). For a realistic efficiency of 0.5, for example, we found that $M=400$ for SLICE, whereas $M=800$ for GAM. In that respect, SLICE can be employed as an useful tool in applications of GAM, especially where the number of available cells is small, such as in the analysis of sample tissues or biopsies.

Summarizing, our findings illustrate how the level of variability of in-silico contact matrices is affected by the number of single cells, $\mathrm{N}$, and by the experimental efficiency, and how different technologies perform in different situations. Overall, under same conditions, SPRITE turns out to be the more 
suitable method to extract pair-wise contact information from small cell samples as $\mathrm{M}$ is smaller than in $\mathrm{Hi}-\mathrm{C}$, and GAM requires a much larger number of cells for replicate robustness. Interestingly, however, at realistic efficiency values for Hi-C, SPRITE and GAM, the number of required cells become similar across the three methods, especially if SLICE is used in combination with GAM.

\section{Noise-to-signal levels vary with genomic distance differently in Hi-C, SPRITE and GAM}

Finally, we investigated the noise-to-signal level of the entries of contact matrices and how it varies with the genomic separation, with the number of cells, $\mathrm{N}$, and with the efficiency of in-silico experiments. For each entry of a contact map, as discussed before, the noise-to-signal ratio is defined as the ratio of the standard deviation, $\sigma$, to mean value, $\mu$, across replicates from experiments in the same conditions. For a given $\mathrm{N}$ and a given efficiency, we observed that the average noise-to-signal ratio, $\langle\sigma / \mu\rangle$, is strongly dependent on genomic distance (Figure 5a). In the Sox9 locus, we found for both $\mathrm{Hi}-\mathrm{C}$ and SPRITE that $\langle\sigma / \mu\rangle$ grows more than one order of magnitude as the genomic separation increases from $50 \mathrm{~kb}$ to $5 \mathrm{Mb}$. In particular, there is a steep increase above the $1 \mathrm{Mb}$ scale. SPRITE has the lowest $\langle\sigma / \mu\rangle$ ratio at genomic scales below the $\mathrm{Mb}$, but interestingly GAM has an overall less varying noise-to-signal level. This is deriving from the GAM methodology that in a single slice samples DNA regions spanning the entire nucleus. Therefore, at larger genomic separations, GAM has almost a one order of magnitude lower noise-to-signal ratio than Hi-C and SPRITE.

At a given genomic distance and efficiency, as expected, the noise-to-signal ratio decreases as the number of cells, $\mathrm{N}$, is increased in our computational experiments (Figure 5b). Consistent with the Central Limit Theorem, it follows an inverse squared power law in $\mathrm{N}$ (i.e., $\mathrm{N}^{-1 / 2}$ ). A consequence of such a scaling behaviour is that single-cell $(\mathrm{N}=1)$ contact maps become highly noisy at large genomic separations. For example, at the $1 \mathrm{Mb}$ scale and for a detection efficiency of 0.5 , the noise-to-signal ratio for $\mathrm{N}=1$ is larger than $100 \%$ for all three methods, $\mathrm{Hi}-\mathrm{C}$ having the largest fluctuations with $\langle\sigma / \mu\rangle>1000 \%$. As expected, the noise-to-signal ratio is also strongly affected by the experimental efficiency (Figure 5c): in brief, we find that for a given genomic distance and for a given $\mathrm{N},\langle\sigma / \mu\rangle$ decreases roughly as an inverse power law of the efficiency in our in-silico study.

In summary, we find that GAM is the method comparatively less noise affected at larger genomic distances, while SPRITE has the best performance below the Mb scale.

\section{DISCUSSION}

$\mathrm{Hi}-\mathrm{C}$, SPRITE and GAM are powerful technologies to generate genomic contact maps, which return different measures of physical proximity between DNA sites, each affected by specific biases and limitations. As we lack an experimental benchmark to compare their performance, in this study we ran a computational investigation where we implemented in-silico the three methods on ensembles of known single-molecule 3D structures to compare them within a simplified, yet fully controlled framework. In particular, we focused, as a case study, on a $6 \mathrm{Mb}$ wide region around the Sox 9 gene in mESC. To check the robustness of our main conclusions, we also investigated a region around the HoxD genes in mESC and around the Epha4 gene in CHLX-12 cells, as well as a toy block-copolymer model, finding analogous results. 
The agreement between independent Hi-C, SPRITE and GAM bulk data and in-silico maps in all the studied loci supports the use of our single-molecule 3D structures and the in-silico method as a proxy for real experiments, providing a tool to compare those technologies in a fair way. Importantly, in all the studied cases, we find that in-silico Hi-C, SPRITE and GAM bulk contact data, as well as SLICE interaction probabilities, all faithfully represent the known spatial conformations of the model polymers. That also suggests that our approach has no biases in favour of any of the three technologies. In particular, we observed that the different methods identify very similar pairwise contact patterns, such as TADs and sub-TADs, which are found to correspond to the known underlying structure of the 3D conformations of the polymer ensemble.

We analysed how the entries of the contact maps of Hi-C, SPRITE and GAM are differently affected by some important parameters of the computational experiments, such as the number of in-silico cells, $\mathrm{N}$, the detection efficiency and the genomic distance. We also quantified how the stochasticity of singlecell experiments is dependent on the intrinsic variability of chromatin 3D conformations. In summary, we showed that if $\mathrm{N}$ is below a threshold value $\mathrm{M}$, replicate experiments can return broadly different outcomes. The value of $\mathrm{M}$, consistent with arguments based on the Central Limit-Theorem, increases as the efficiency of the experiment decreases. For equal conditions, $M$ is different in different technologies: SPRITE is the method having the lowest $M$ and so better performing on samples with a small number of cells; GAM has the highest value, but in combination with SLICE M is drastically reduced. In real applications, it is important to take into account that the efficiency is different across the three methods, and the corresponding values of $\mathrm{M}$ can become similar. For example, the experimental estimations of the efficiency of $\mathrm{Hi}-\mathrm{C}$ is around $0.05^{50}$, SPRITE is around 0.05 too (M. Guttmann personal communication) and for GAM around $0.5^{20}$ : in those considitions we find that $\mathrm{M}$ is around 650, 250 and 800 for respectively Hi-C, SPRITE and GAM; additionally, when GAM is combined with SLICE, M becomes approximately 400. Reassuringly, we found that the use of large $\mathrm{N}$ can generally compensate for a limited efficiency and in the bulk limit the different technologies are all overall faithful to the benchmark model 3D structures. Finally, we analysed the noise-to-signal ratio in contact maps as a function of the genomic distance and found that GAM is less noise sensitive at large genomic separations, while $\mathrm{Hi}-\mathrm{C}$ and SPRITE at lower distances, under the same conditions.

More generally, the consistent behaviour of our computational analyses across all the investigated cases (models of real loci as well as toy models) supports the view that their conclusions have a broad validity and can help guiding the design of novel Hi-C, SPRITE and GAM experiments in different contexts and applications. 


\section{ACKNOWLEDGEMENTS}

M.N. acknowledges support from CINECA ISCRA ID HP10CYFPS5 and HP10CRTY8P, Einstein BIH Fellowship Award (EVF-BIH-2016-282), Regione Campania SATIN Project 2018-2020, and computer resources from INFN, CINECA, ENEA CRESCO/ENEAGRID ${ }^{57}$ and Scope/ReCAS at the University of Naples. A.P., R.K., A.K., I.I.A thank the Helmholtz Association (Germany) for support. M.N. and A.P. acknowledge support from the National Institutes of Health Common Fund 4D Nucleome Program grant U54DK107977 and the EU H2020 Marie Curie ITN n.813282. I.I.A. acknowledges support from the Federation of European Biochemical Societies (FEBS Long-Term Fellowship).

\section{AUTHOR CONTRIBUTIONS}

M.N. designed the project with inputs from A.P.. M.N., L.F. and F.M. developed the modelling. L.F. and F.M. ran computer simulations and performed data analyses with help from S.B., A.M.C., A.E. and M.C.. R.K., A.K. and I.I.A. produced and normalized the GAM dataset. M.N., L.F., F.M. and A.P. wrote the manuscript, with inputs from all the authors.

\section{REFERENCES}

1. Bickmore, W. A. The Spatial Organization of the Human Genome. Annu. Rev. Genomics Hum. Genet. 14, 67-84 (2013).

2. Dekker, J. \& Misteli, T. Long-range chromatin interactions. Cold Spring Harb. Perspect. Biol. 7, a019356 (2015).

3. Pombo, A. \& Dillon, N. Three-dimensional genome architecture: Players and mechanisms. Nature Reviews Molecular Cell Biology 16, 245-257 (2015).

4. Dekker, J. \& Mirny, L. The 3D Genome as Moderator of Chromosomal Communication. Cell 164, 1110-1121 (2016).

5. Dixon, J. R., Gorkin, D. U. \& Ren, B. Chromatin Domains: The Unit of Chromosome Organization. Molecular Cell 62, 668-680 (2016).

6. Spielmann, M., Lupiáñez, D. G. \& Mundlos, S. Structural variation in the 3D genome. Nature Reviews Genetics 19, 453-467 (2018).

7. Finn, E. H. \& Misteli, T. Molecular basis and biological function of variability in spatial genome organization. Science 365, eaaw9498 (2019).

8. Kempfer, R. \& Pombo, A. Methods for mapping 3D chromosome architecture. Nature Reviews Genetics 21, 207-226 (2019).

9. Cattoni, D. I. et al. Single-cell absolute contact probability detection reveals chromosomes are organized by multiple low-frequency yet specific interactions. Nat. Commun. 8, 1-10 (2017).

10. Bintu, B. et al. Super-resolution chromatin tracing reveals domains and cooperative 
interactions in single cells. Science (80-. ). 362, eaau1783 (2018).

11. Cardozo Gizzi, A. M. et al. Microscopy-Based Chromosome Conformation Capture Enables Simultaneous Visualization of Genome Organization and Transcription in Intact Organisms. Mol. Cell 74, 212-222.e5 (2019).

12. Finn, E. H. et al. Extensive Heterogeneity and Intrinsic Variation in Spatial Genome Organization. Cell 176, 1502-1515.e10 (2019).

13. Lieberman-Aiden, E. et al. Comprehensive mapping of long-range interactions reveals folding principles of the human genome. Science (80-. ). 326, 289-293 (2009).

14. Fullwood, M. J. et al. An oestrogen-receptor- $\alpha$-bound human chromatin interactome. Nature 462, 58-64 (2009).

15. Rao, S. S. P. et al. A 3D map of the human genome at kilobase resolution reveals principles of chromatin looping. Cell 159, 1665-1680 (2014).

16. Mumbach, M. R. et al. HiChIP: Efficient and sensitive analysis of protein-directed genome architecture. Nat. Methods 13, 919-922 (2016).

17. Oudelaar, A. M. et al. Single-allele chromatin interactions identify regulatory hubs in dynamic compartmentalized domains. Nat. Genet. 50, 1744-1751 (2018).

18. Hsieh, T. H. S. et al. Mapping Nucleosome Resolution Chromosome Folding in Yeast by Micro-C. Cell 162, 108-119 (2015).

19. Krietenstein, N. et al. Ultrastructural details of mammalian chromosome architecture. bioRxiv 639922 (2019). doi:10.1101/639922

20. Beagrie, R. A. et al. Complex multi-enhancer contacts captured by genome architecture mapping. Nature 543, 519-524 (2017).

21. Quinodoz, S. A. et al. Higher-Order Inter-chromosomal Hubs Shape 3D Genome Organization in the Nucleus. Cell 174, 744-757.e24 (2018).

22. Nora, E. P. et al. Spatial partitioning of the regulatory landscape of the X-inactivation centre. Nature 485, 381-385 (2012).

23. Dixon, J. R. et al. Topological domains in mammalian genomes identified by analysis of chromatin interactions. Nature 485, 376-380 (2012).

24. Fraser, J. et al. Hierarchical folding and reorganization of chromosomes are linked to transcriptional changes in cellular differentiation. Mol. Syst. Biol. 11, 852 (2015).

25. Chiariello, A. M., Annunziatella, C., Bianco, S., Esposito, A. \& Nicodemi, M. Polymer physics of chromosome large-scale 3D organisation. Sci. Rep. 6, 29775 (2016).

26. Bianco, S. et al. Modeling Single-Molecule Conformations of the HoxD Region in Mouse Embryonic Stem and Cortical Neuronal Cells. Cell Rep. 28, 1574-1583.e4 (2019).

27. Bianco, S. et al. Polymer physics predicts the effects of structural variants on chromatin architecture. Nat. Genet. 50, 662-667 (2018).

28. Lupiáñez, D. G. et al. Disruptions of topological chromatin domains cause pathogenic rewiring 
of gene-enhancer interactions. Cell 161, 1012-1025 (2015).

29. Andrey, G. et al. A switch between topological domains underlies HoxD genes collinearity in mouse limbs. Science (80-. ). 340, 1234167 (2013).

30. Noordermeer, D. et al. The dynamic architecture of Hox gene clusters. Science (80-. ). 334, 222225 (2011).

31. Li, Q. et al. The three-dimensional genome organization of Drosophila melanogaster through data integration. Genome Biol. 18, 145 (2017).

32. Serra, F. et al. Automatic analysis and 3D-modelling of Hi-C data using TADbit reveals structural features of the fly chromatin colors. PLoS Comput. Biol. 13, e1005665 (2017).

33. Nir, G. et al. Walking along chromosomes with super-resolution imaging, contact maps, and integrative modeling. PLoS Genet. 14, e1007872 (2018).

34. Lin, D., Bonora, G., Yardimci, G. G. \& Noble, W. S. Computational methods for analyzing and modeling genome structure and organization. Wiley Interdiscip. Rev. Syst. Biol. Med. 11, e1435 (2018).

35. Buckle, A., Brackley, C. A., Boyle, S., Marenduzzo, D. \& Gilbert, N. Polymer Simulations of Heteromorphic Chromatin Predict the 3D Folding of Complex Genomic Loci. Mol. Cell 72, 786797.e11 (2018).

36. Fiorillo, L. et al. A modern challenge of polymer physics: Novel ways to study, interpret, and reconstruct chromatin structure. Wiley Interdisciplinary Reviews: Computational Molecular Science e1454 (2019).

37. Nicodemi, M. \& Prisco, A. Thermodynamic pathways to genome spatial organization in the cell nucleus. Biophys. J. 96, 2168-2177 (2009).

38. Bohn, M. \& Heermann, D. W. Diffusion-driven looping provides a consistent provides a consistent framework for chromatin organization. PLoS One 5, e12218 (2010).

39. Barbieri, M. et al. Complexity of chromatin folding is captured by the strings and binders switch model. Proc. Natl. Acad. Sci. U. S. A. 109, 16173-16178 (2012).

40. Brackley, C. A., Taylor, S., Papantonis, A., Cook, P. R. \& Marenduzzo, D. Nonspecific bridginginduced attraction drives clustering of DNA-binding proteins and genome organization. Proc. Natl. Acad. Sci. U. S. A. 110, E3605-E3611 (2013).

41. Jost, D., Carrivain, P., Cavalli, G. \& Vaillant, C. Modeling epigenome folding: formation and dynamics of topologically associated chromatin domains. Nucleic Acids Res. 42, 9553-61 (2014).

42. Sanborn, A. L. et al. Chromatin extrusion explains key features of loop and domain formation in wild-type and engineered genomes. Proc. Natl. Acad. Sci. U. S. A. 112, E6456-E6465 (2015).

43. Fudenberg, G. et al. Formation of Chromosomal Domains by Loop Extrusion. Cell Rep. 15, 20382049 (2016).

44. Di Pierro, M., Zhang, B., Aiden, E. L., Wolynes, P. G. \& Onuchic, J. N. Transferable model for chromosome architecture. Proc. Natl. Acad. Sci. 113, 12168-12173 (2016). 
45. Fiorillo, L. et al. Inference of chromosome 3D structures from GAM data by a physics computational approach. Methods S1046-2023(18)30485-7 (2019).

46. Barbieri, M. et al. Active and poised promoter states drive folding of the extended HoxB locus in mouse embryonic stem cells. Nat. Struct. Mol. Biol. 24, 515-524 (2017).

47. Kragesteen, B. K. et al. Dynamic 3D chromatin architecture contributes to enhancer specificity and limb morphogenesis. Nat. Genet. 50, 1463-1473 (2018).

48. Yang, T. et al. HiCRep: assessing the reproducibility of Hi-C data using a stratum-adjusted correlation coefficient. Genome Res. 27, 1939-1949 (2017).

49. Dekker, J. et al. The 4D nucleome project. Nature 549, 219-226 (2017).

50. Nagano, T. et al. Single-cell Hi-C reveals cell-to-cell variability in chromosome structure. Nature 502, 59-64 (2013).

51. Nagano, T. et al. Cell-cycle dynamics of chromosomal organization at single-cell resolution. Nature 547, 61-67 (2017).

52. Flyamer, I. M. et al. Single-nucleus Hi-C reveals unique chromatin reorganization at oocyte-tozygote transition. Nature 544, 110-114 (2017).

53. Stevens, T. J. et al. 3D structures of individual mammalian genomes studied by single-cell Hi-C. Nature 544, 59-64 (2017).

54. Ramani, V. et al. Massively multiplex single-cell Hi-C. Nat. Methods 14, 263-266 (2017).

55. Lando, D., Stevens, T. J., Basu, S. \& Laue, E. D. Calculation of 3D genome structures for comparison of chromosome conformation capture experiments with microscopy: An evaluation of single-cell Hi-C protocols. Nucleus 9, 190-201 (2018).

56. Díaz, N. et al. Chromatin conformation analysis of primary patient tissue using a low input Hi-C method. Nat. Commun. 9, 1-13 (2018).

57. Ponti, G. et al. The role of medium size facilities in the HPC ecosystem: The case of the new CRESCO4 cluster integrated in the ENEAGRID infrastructure. in Proceedings of the 2014 International Conference on High Performance Computing and Simulation, HPCS 2014 10301033 (2014). 


\section{MAIN FIGURES}

a)

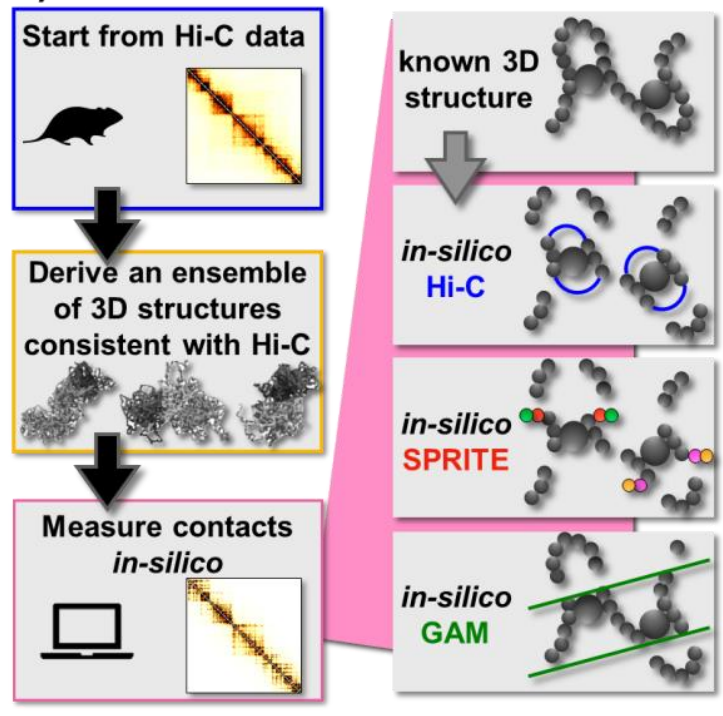

b)

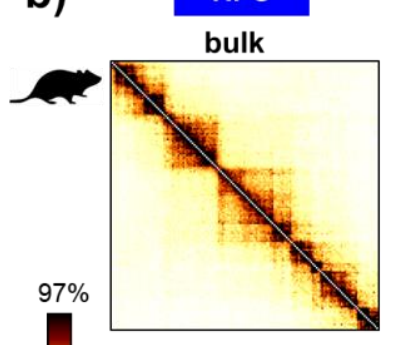

$r_{s}=0.83$

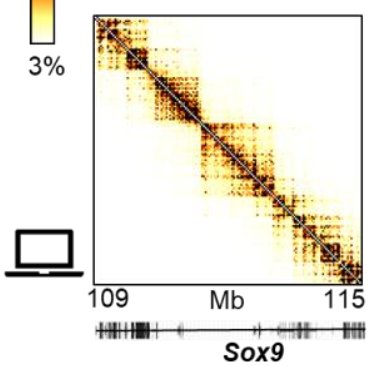

SPRITE

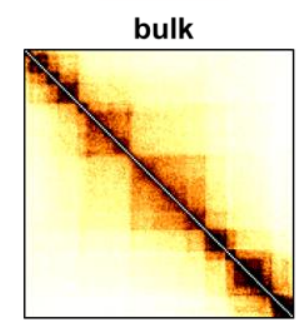

$r_{\mathrm{s}}=0.92$

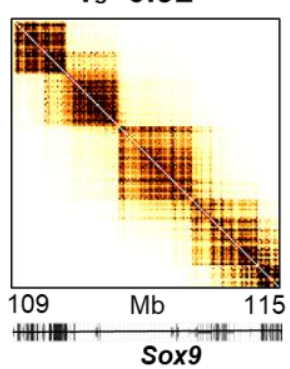

GAM

1122 cells

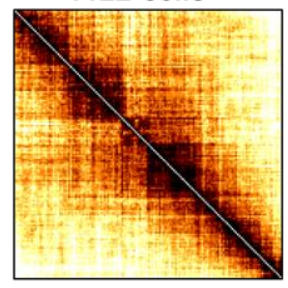

$r_{s}=0.79$

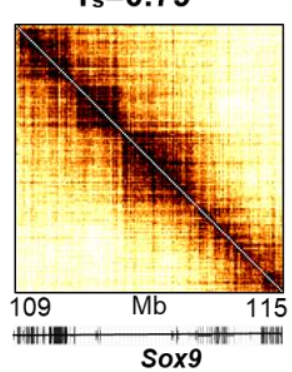

Figure 1. In-silico Hi-C, SPRITE and GAM average contact maps match experimental data.

a) By use of the PRISMR procedure ${ }^{27}$, from $\mathrm{Hi}-\mathrm{C}$ data the polymer model of the DNA locus of interest is inferred and, based on polymer-physics, an ensemble of its single-molecule 3D conformations, consistent with the input $\mathrm{Hi}-\mathrm{C}$ data, derived. We implemented computationally the Hi-C, SPRITE and GAM methods on those 3D structures and measure in-silico the corresponding contact maps.

b) Albeit inferred from $\mathrm{Hi}-\mathrm{C}$ data only, the model 3D conformations return average contact maps (bottom) well matching the Hi-C and the independent SPRITE and GAM experimental data (top) in the case study of the Sox9 locus (chr11:109Mb-115Mb, mm9) in mESC. Experimental Hi-C and SPRITE maps are bulk data 21,23 , while GAM data are from a new dataset constructed from 1122 F123 cells (Materials and Methods), and correspondingly the in-silico maps. The color scale represents the percentiles of each dataset. The Spearman correlation coefficients $\left(r_{s}\right)$ between model and experiment are reported in the middle, as Pearson or HiCRep correlations have similar values (Supplementary Table 1a). 


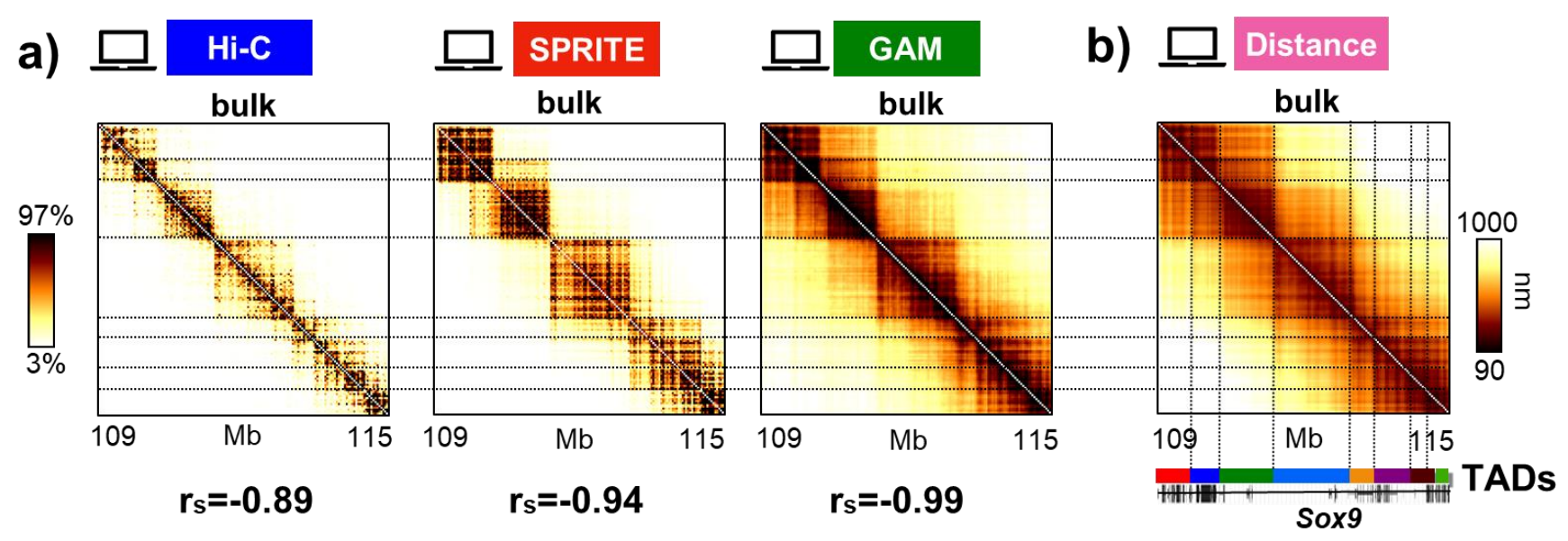

Figure 2. Bulk Hi-C, SPRITE and GAM data are all overall faithful to the average 3D distances.

a) The in-silico bulk Hi-C, SPRITE and GAM maps of the Sox9 locus, although corresponding to different measures of DNA physical proximity, return similar contact patterns. The color scale represents the percentiles of each contact map. The Spearman correlation coefficients are reported, on the bottom, between each contact map and the average 3D distance matrix in panel b) of the known single-molecule 3D conformations of the locus model (Pearson and HiCRep return similarly high correlations, Supplementary Table 1b).

b) The average 3D distance map derived from the ensemble of in-silico model single-molecule 3D conformations is shown. Its high correlation values with each of three contact maps of panel a) (Supplementary Table 1b) illustrates that the three technologies faithfully capture the average pairwise distances of the system. We find that the TADs of the locus ${ }^{23}$ (different colors of the bar in the bottom) correspond to the domain-like patterns of the average distance matrix. They are well identified by the in-silico Hi-C, SPRITE and GAM bulk maps, as highlighted by the drawn vertical and horizontal lines. In particular, GAM captures well the longer-range inter-TADs contacts. 
bioRxiv preprint doi: https://doi.org/10.1101/2020.04.24.059915; this version posted April 25, 2020. The copyright holder for this preprint (which was not certified by peer review) is the author/funder, who has granted bioRxiv a license to display the preprint in perpetuity. It is made

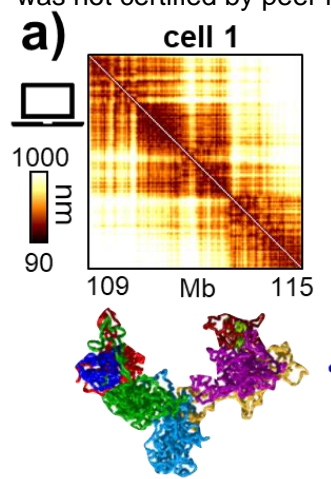
cell 2 vailable under acefl $\mathrm{B}_{3}$-NC-ND 4.0 Internatioballicense.
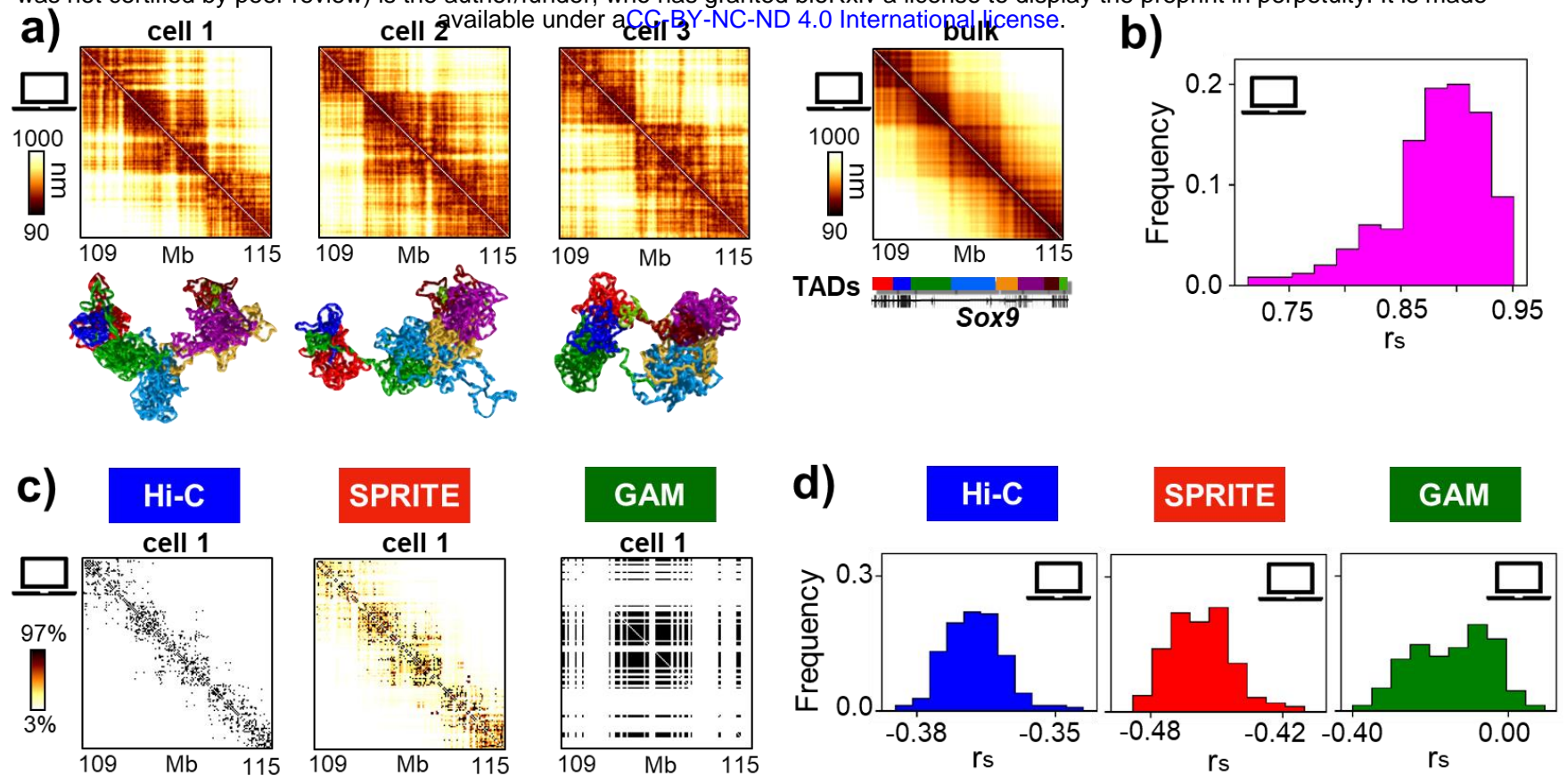

Figure 3. Stochasticity of single-cell contact maps reflects the intrinsic variability of single-molecule 3D conformations.

a) The variability of single-molecule conformations of the model of the Sox9 locus is represented here by three examples (bottom, the color scheme reflects the colors of the TADs of the locus ${ }^{23}$, shown in the color bar). Their corresponding in-silico single-cell distance maps (on top) can be different from the average distance matrix (left). For example, the Spearman correlations between the shown in-silico single-cell and the average distance maps are: $r_{s}=0.89$ for cell $1 ; r_{s}=0.82$ for cell $2 ; r_{s}=0.83$ for cell 3 . Replicate single-cell experiments can differ from each other just because they capture distinct underlying chromatin structures.

b) The Spearman correlation between in-silico single-cell distance maps and the average distance map has a broad distribution (mean value $r_{S}=0.88$ and median $r_{S}=0.89$, Materials and Methods). Mean Pearson and HiCRep correlations are analogous and reported in Supplementary Table 1c.

c) The in-silico single-cell Hi-C, SPRITE and GAM contact maps corresponding to the first of the three insilico cells of panel a) are shown (color scale indicates the percentiles of each map). Here, the in-silico efficiency is set to 1 , so all contacts are captured in Hi-C or SPRITE, and all segregated windows are detected in GAM.

d) The distribution is shown of Spearman correlation coefficients between in-silico single-cell contact matrices at efficiency 1 and their corresponding in-silico single-cell distance matrices. In-silico singlecell data average correlations (Supplementary Table 1d,e and Materials and Methods) are overall significantly lower than correlations in bulk data (Figure $\mathbf{2}$ and Supplementary Table 1b). Single-cell Hi$\mathrm{C}$ and SPRITE perform comparatively better than single-cell GAM in reproducing the pattern of physical distances, as single-cell GAM derives from only a single slice cut out of a nucleus. Overall, single-cell contact matrices are less faithful to their corresponding distance map than bulk matrices (see Figure 2). Here we considered the ideal case where the efficiency is 1 to highlight the effects on contact data of the variability of single molecules, yet in real experiments the efficiency is different in the three technologies and well below 1 (see Main Text and Figure 4). 

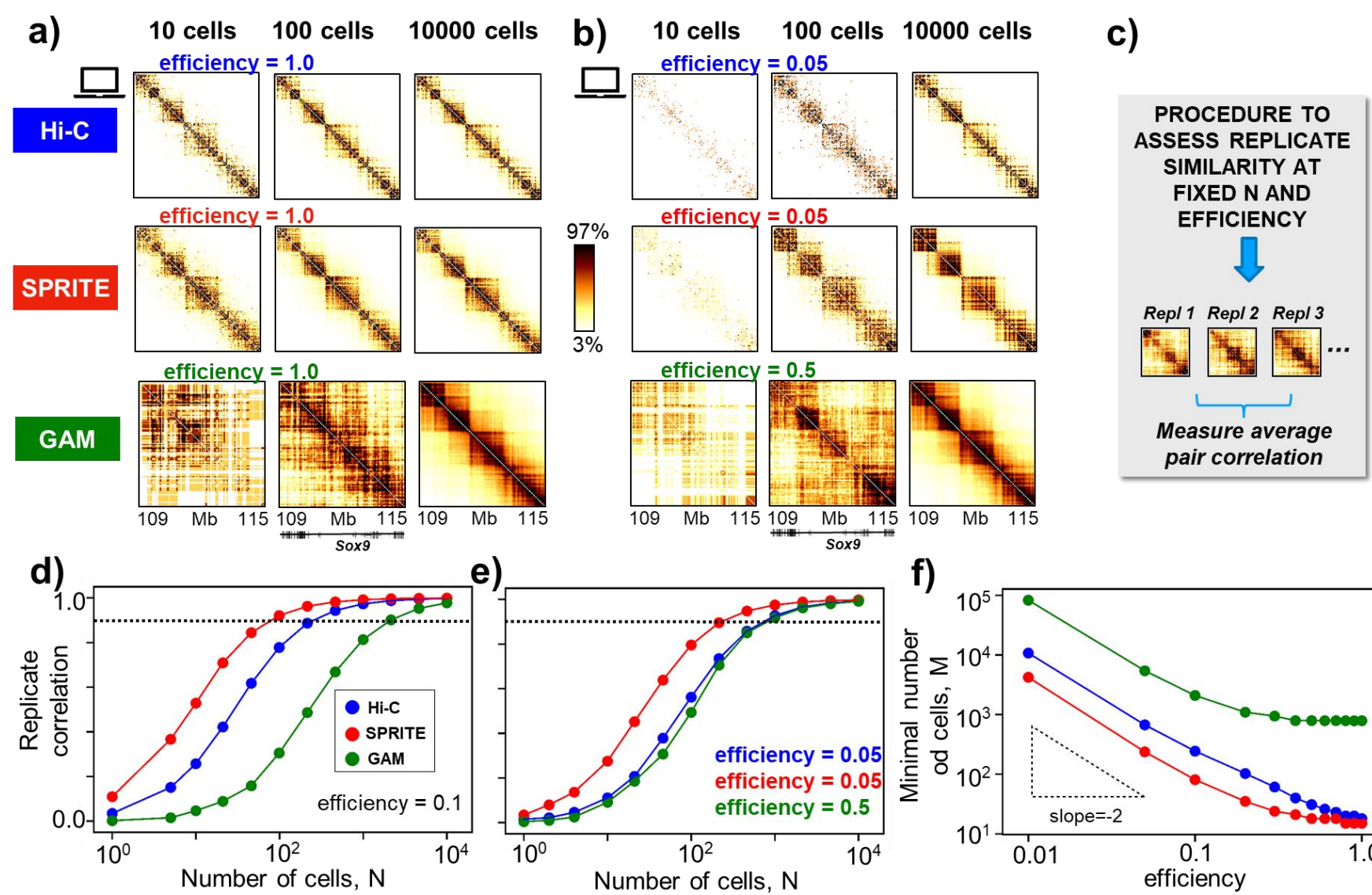

e)

Figure 4. Threshold cell number required for replicate reproducibility differs in Hi-C, SPRITE and GAM.

a) The in-silico Hi-C, SPRITE and GAM contact maps of the Sox9 locus depend on the number of in-silico cells, $\mathrm{N}$, considered in the experiment (here is shown the case with efficiency equal to 1 ), albeit in the bulk limit (large N), the effects of cell-to-cell variability are averaged out for all the three technologies. Color scale indicates the percentiles of each map.

b) Results analogous to those in panel a) are shown in the case where efficiencies similar to those found in real experiments are considered: here, for $\mathrm{Hi}$-C and SPRITE the in-silico efficiency is set equal to 0.05 , and for GAM equal to 0.5 (see Main Text).

c) To assess the similarity between in-silico replicate contact maps (i.e. maps obtained with same number of cells, $\mathrm{N}$, and efficiency), we measured the average Pearson correlation between them. The minimal number of cells, $\mathrm{M}$, to have a reproducible output map is defined as the value of $\mathrm{N}$ where the average Pearson correlation between replicates crosses the threshold $r_{t}=0.9$ (Main Text and Materials and Methods).

d) The Pearson correlation is shown between replicate experiments as a function of $\mathrm{N}$ for Hi-C, SPRITE and GAM at a given efficiency (0.1). The dashed line is the threshold correlation value $r_{t}=0.9$. Analogous results are found when the Spearman or HiCRep correlations are considered (Supplementary Figure 5). 
e) Results analogous to those in panel d) are shown in the case of efficiencies similar to those in real experiments, as discussed in panel b). As GAM has a higher efficiency, its corresponding behavior with $\mathrm{N}$ becomes closer to those of $\mathrm{Hi}-\mathrm{C}$ and SPRITE. For example, here, for the reported realistic efficiencies of 0.05 for Hi-C and SPRITE, and 0.5 for GAM, M is respectively approximately 650, 250 and 800.

f) The value of $M$ is shown for Hi-C, SPRITE and GAM as a function of the efficiency. $M$ increases as the efficiency is reduced and grows approximately as an inverse squared power law at small efficiencies. The obtained values of $\mathrm{M}$ are consistent with the Central Limit Theorem (Materials and Methods). For a given efficiency, $\mathrm{M}$ is the smallest in SPRITE, a factor of two higher in $\mathrm{Hi}-\mathrm{C}$ and one order of magnitude higher in GAM. 

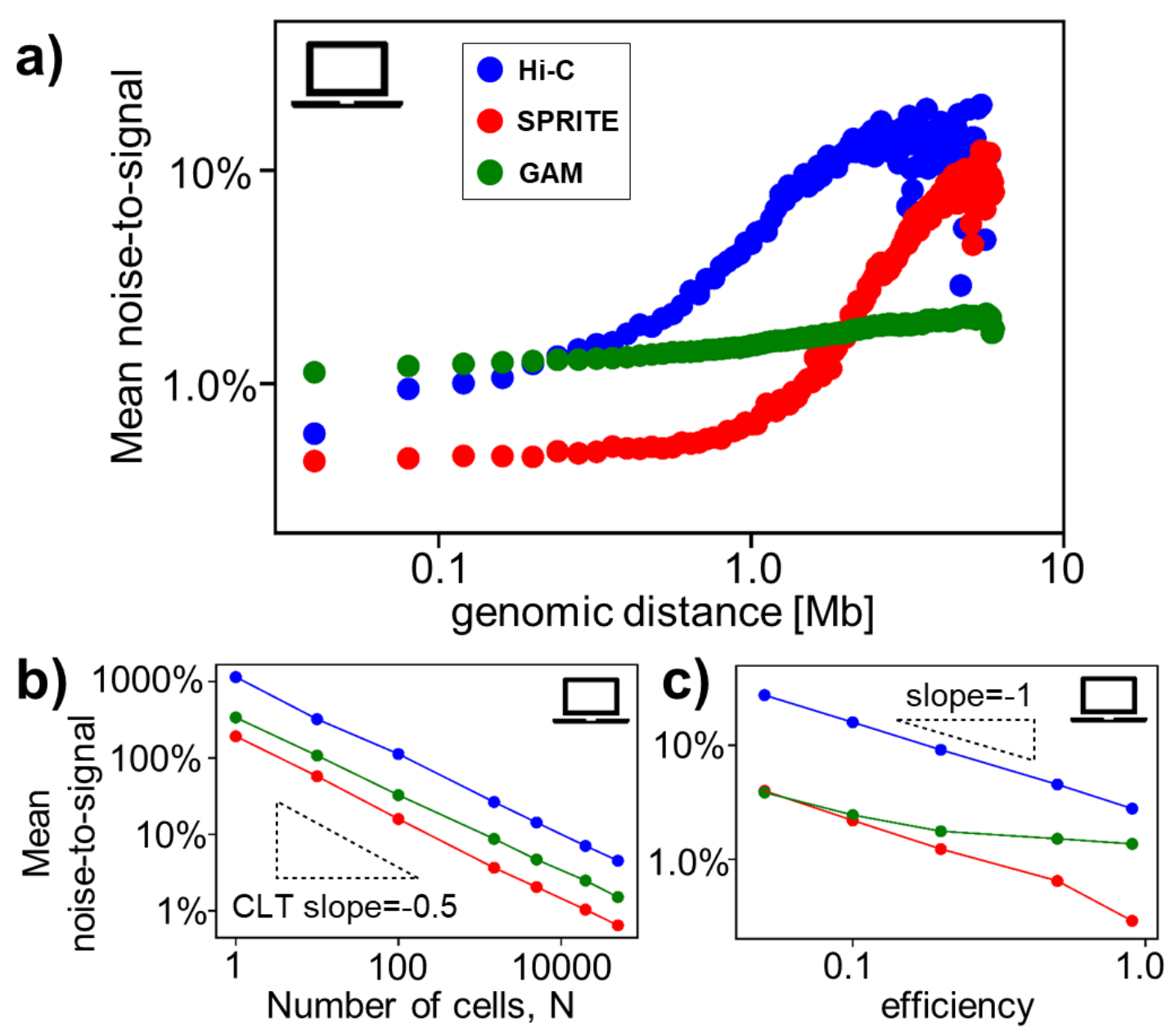

Figure 5. Noise-to-signal levels vary with genomic distance differently in Hi-C, SPRITE and GAM.

a) The mean noise-to-signal ratio $\langle\sigma / \mu\rangle$ of a contact map (see Main Text and Materials and Methods), for a given number of cells $\mathrm{N}$ and efficiency, depends on the considered genomic separation. For the Sox9 locus, in Hi-C and SPRITE, the noise-to-signal ratio drastically grows above $1 \mathrm{Mb}$, i.e. the scale of TADs, while GAM retains low noise-to-signal ratios across larger genomic separations (the case shown is for $\mathrm{N}=50000$ and efficiency $=0.5$ ).

b) For a given genomic distance and efficiency (the case shown is for $1 \mathrm{Mb}$ and efficiency=0.5), $\langle\sigma / \mu\rangle$ decreases with $\mathrm{N}$ as an inverse square root, as expected from the Central Limit Theorem.

c) Analogously, for a given genomic distance and $\mathrm{N}$ (the case shown is for $1 \mathrm{Mb}$ and $\mathrm{N}=50000),\langle\sigma / \mu\rangle$ increases, for small efficiencies, approximately as an inverse power law when the efficiency is reduced. 


\section{SUPPLEMENTARY FIGURES}

a)

\section{Hi-C}

bulk

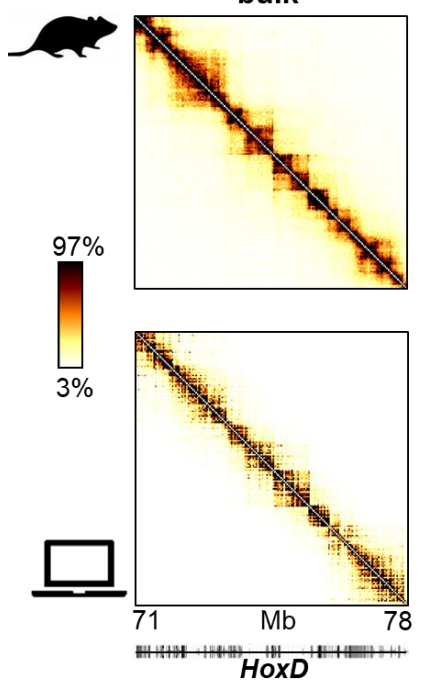

SPRITE

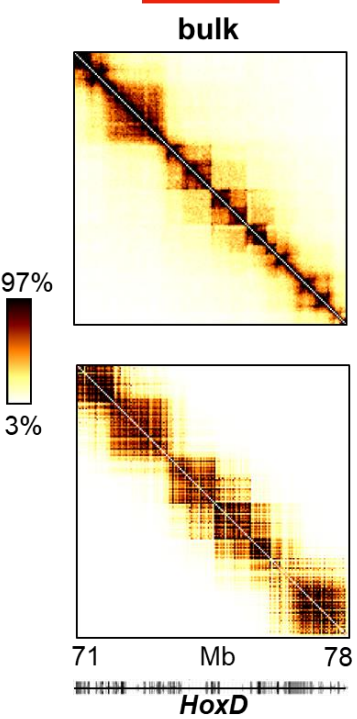

GAM

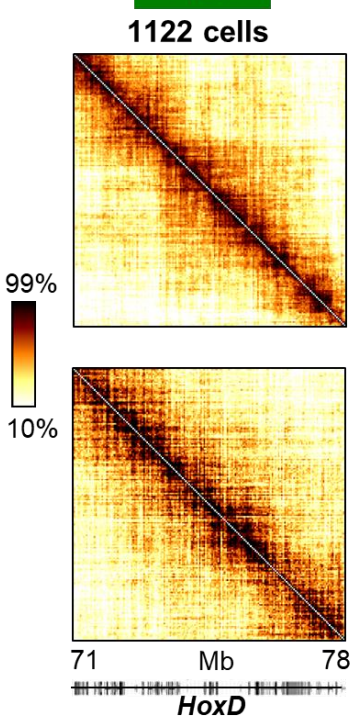

b)
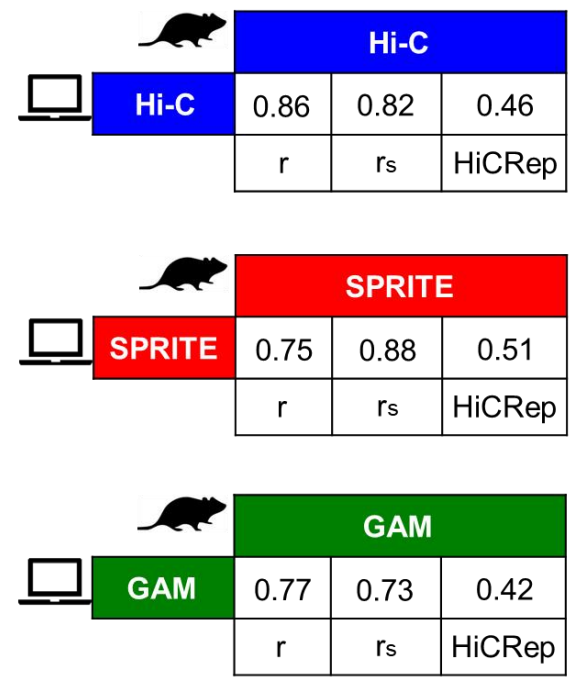

Supplementary Figure 1. In-silico average contact maps of the HoxD locus compare well with Hi-C, SPRITE and GAM experimental data.

a) In the case of the murine HoxD locus (chr2:71Mb-78Mb, mm9) in mESC, our 3D conformations return in-silico contact maps (bottom) that match well with $\mathrm{Hi}-\mathrm{C}^{23}$, SPRITE ${ }^{21}$ and GAM experimental data from mESC (top). GAM data are from the new dataset of 1122 nuclear slices (Materials and Methods). Correspondingly, in-silico Hi-C and SPRITE are bulk data, while in-silico GAM data are from 1122 in-silico cells (see Materials and Methods). Color scale indicates the percentiles of the maps.

b) Pearson, Spearman and HiCRep correlations between the in silico and experimental maps of panel a), for the three technologies. 


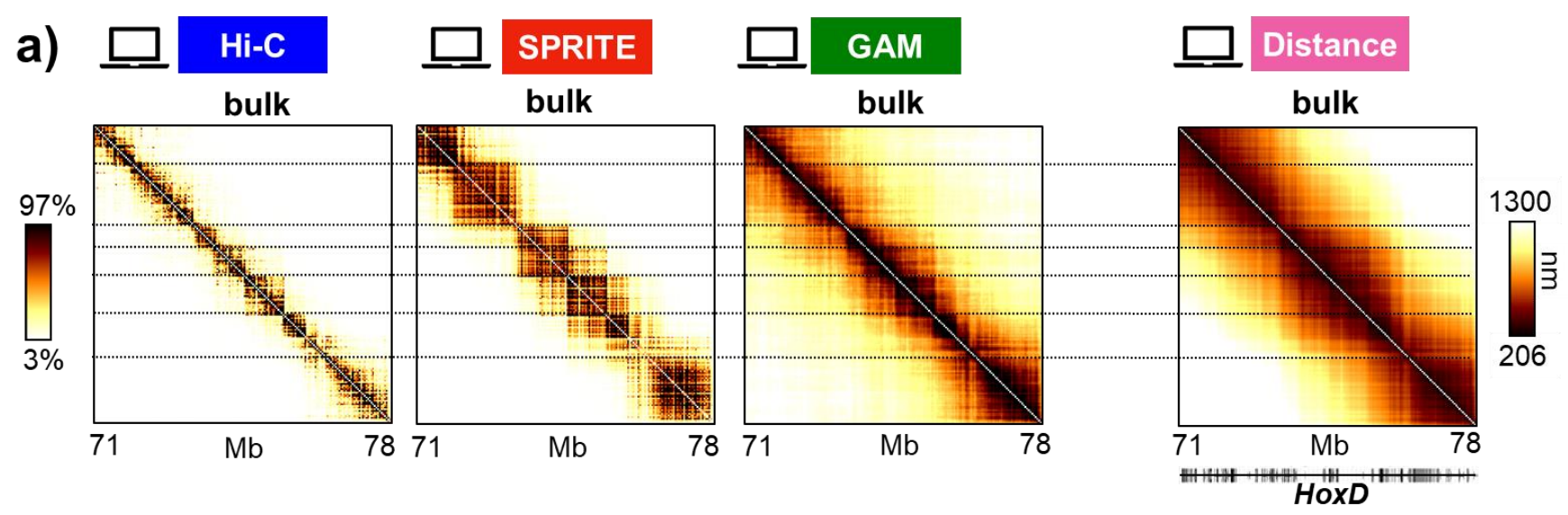

\begin{tabular}{c|c|c|c|} 
b) $\frac{\square}{\text { bulk }}$ & \multicolumn{3}{|c|}{ Distance } \\
\cline { 2 - 4 } Hi-C & -0.48 & -0.85 & -0.71 \\
\hline SPRITE & -0.69 & -0.90 & -0.61 \\
\hline GAM & -0.91 & -0.99 & -0.78 \\
\hline & $r$ & rs & HiCRep \\
\cline { 2 - 4 } & & &
\end{tabular}

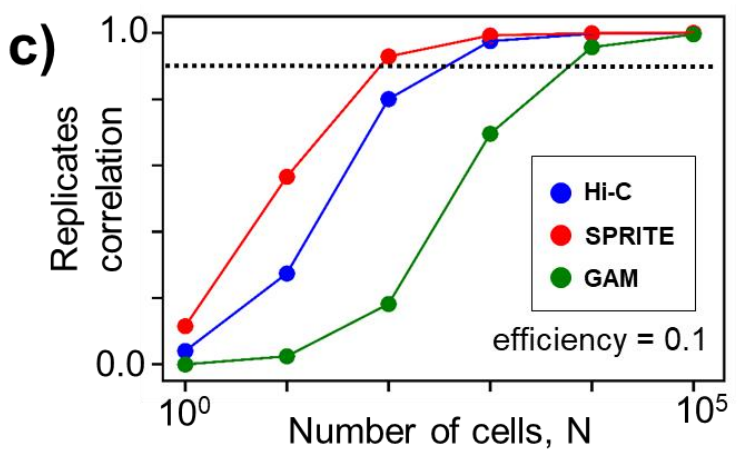

Supplementary Figure 2. In-silico bulk contact maps overall reproduce the average distance map in the HoxD locus.

a) In-silico bulk Hi-C, SPRITE and GAM maps of the HoxD locus return contact patterns that are compatible with the average distance pattern derived from the ensemble of single-molecule 3D conformations. The horizontal lines are drawn to mark the domain-like structure of the distance map. The color scale indicates the percentiles.

b) Pearson, Spearman and HiCRep correlations are reported between the bulk contact maps and the average distance map. Correlations indicate a high degree of similarity, showing that the three technologies all faithfully capture the underlying conformations of the HoxD locus, analogously to the Sox9 case study (Figure 2 and Supplementary Table 1b).

c) The Pearson correlation is shown between replicates against the number of in-silico cells for the HoxD locus at efficiency 0.1. The dashed line is the threshold correlation value $r_{t}=0.9$. The resulting trend is similar to the Sox9 locus case in Figure $4 \mathbf{d}$. Also, the M values found for Hi-C, SPRITE and GAM are analogous to the Sox9 ones in Figure $\mathbf{4 d}$. 

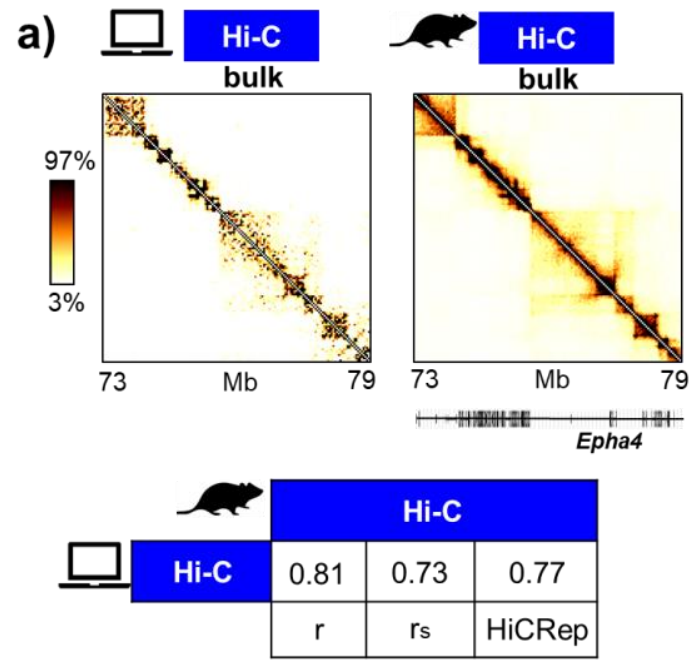

c)

\begin{tabular}{|c|c|c|c|}
\hline$\overline{\text { bulk }}$ & \multicolumn{3}{|c|}{ Distance } \\
\hline $\mathrm{Hi}-\mathrm{C}$ & -0.22 & -0.65 & -0.72 \\
\hline SPRITE & -0.45 & -0.83 & -0.77 \\
\hline GAM & -0.90 & -0.98 & -0.89 \\
\hline & $r$ & rs & HiCRep \\
\hline
\end{tabular}

d)

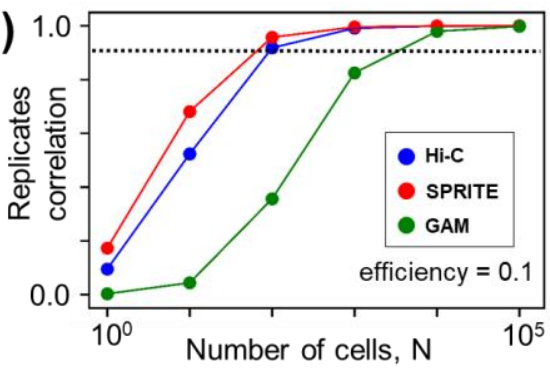

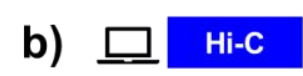

b) $\square \frac{\text { Hi-C }}{\text { bulk }}$

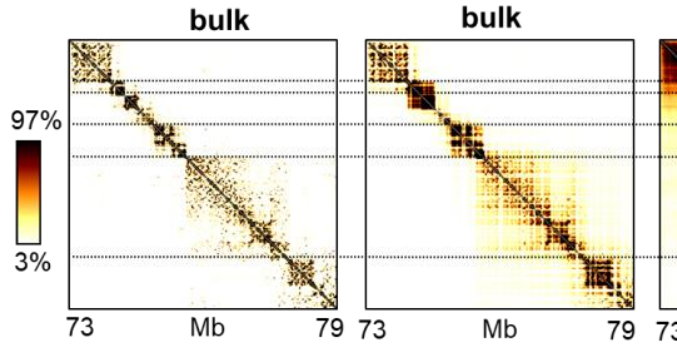

\section{SPRITE

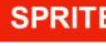

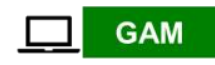

bulk
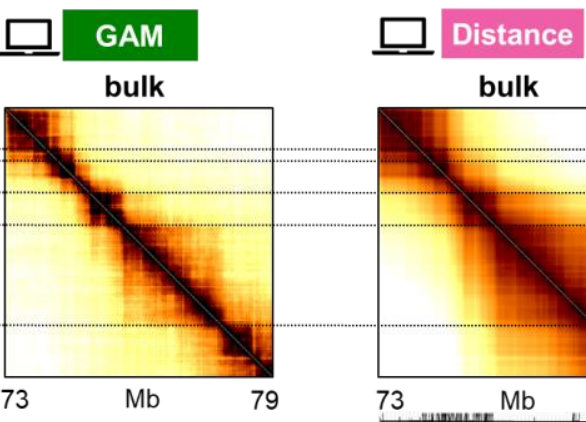

bulk

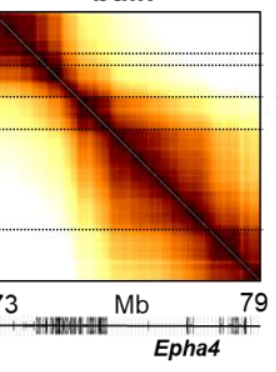

1000

马

50

\section{Supplementary Figure 3. In-silico contact maps from 3D conformations of the Epha4 locus.}

a) In the model of the Epha4 locus in mouse CHLX-12 cells the in-silico bulk Hi-C map (left) is compared to the experimental map ${ }^{15}$ (right) (color scale indicates the percentiles of the maps). In the table on the bottom, Pearson, Spearman and HiCRep correlations are reported, indicating good similarity between the two matrices.

b) The in-silico bulk contact maps are compared with the average distance pattern obtained from the ensemble of 3D conformations of the model of the locus. The horizontal lines are drawn to mark the domain-like structure of the distance map.

c) Pearson, Spearman and HiCRep correlations are reported between each bulk contact map and the average distance map, indicating overall a good degree of similarity for each of the technologies.

d) The Pearson correlation is shown between replicates against the number of in-silico cells for the Epha4 locus, at efficiency 0.1. The dashed line is the threshold correlation value $r_{t}=0.9$. The values of $M$ are compatible with the ones we find in the Sox9 and HoxD loci (Figure 4d and Supplementary Figure 2c), for all three technologies. 
a)

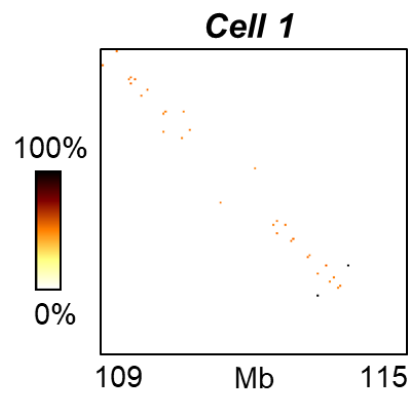

Cell 2

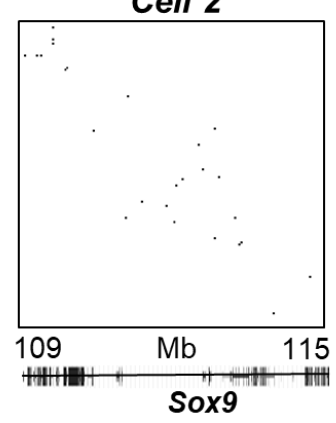

Single cell Hi-C

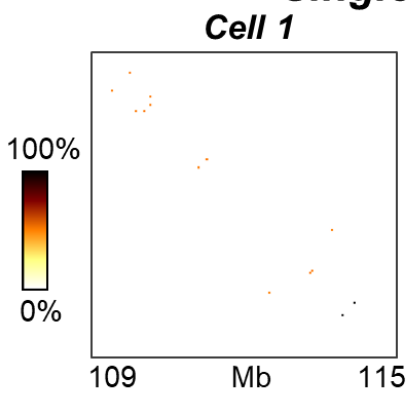

Cell 2

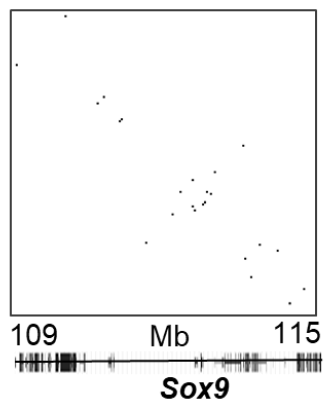

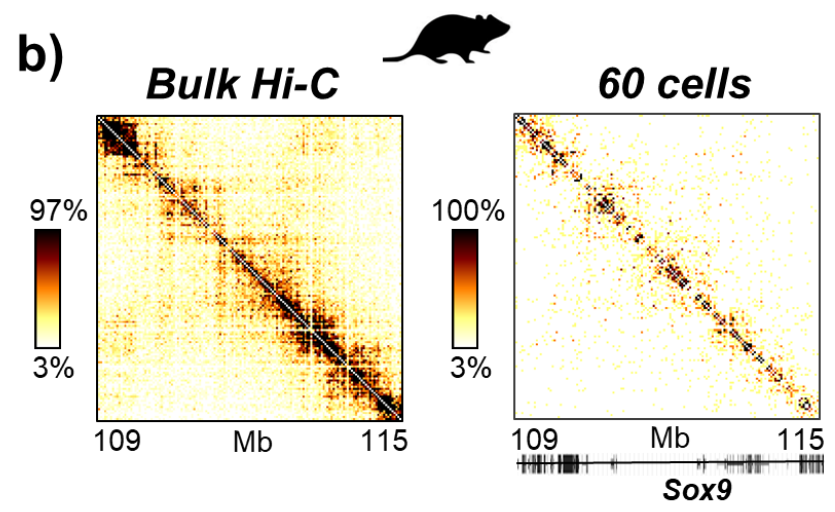

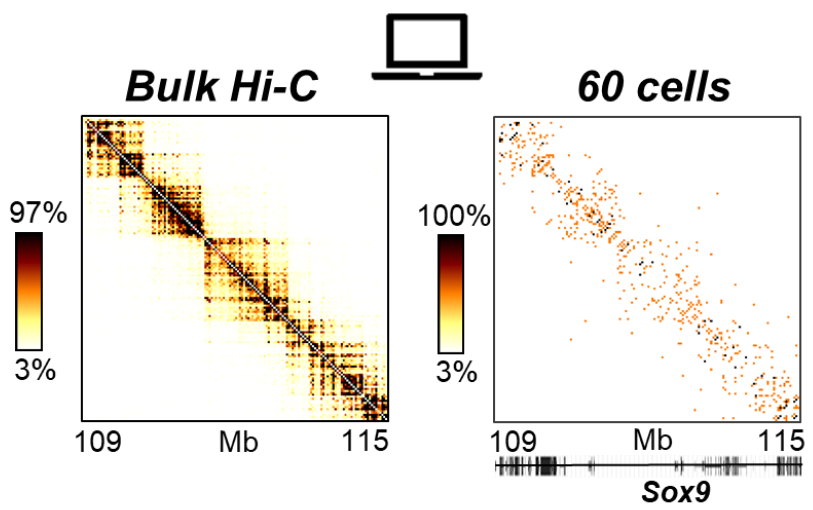

Supplementary Figure 4. Experimental and in-silico single-cell Hi-C data.

a) Left panel. Two examples of experimental single-cell Hi-C contact maps ${ }^{50}$, for the Sox 9 locus in the mouse $C D 4 T_{H} 1$ cells. The mean Spearman correlation between all the available pairs of such single-cell Sox9 maps ${ }^{50}$ is $r_{S}=0.01$ (see also Materials and Methods). Right panel. Two examples of the in-silico single-cell Hi-C maps for the Sox9 locus in mESC. Mean Spearman correlation is $r_{S}=0.01$, consistent to the experimental result (Materials and Methods). The efficiency is set to $0.025^{50}$. Color scale indicates the percentiles of the maps.

b) Left panel. In the same genomic region in CD4 $T_{H} 1$ cells, the average experimental map resulting from 60 available single-cell contact data is compared against the bulk Hi-C map ${ }^{50}$ : their Spearman correlation is $r_{S}=0.33$ (see Materials and Methods). Right panel. A similar calculation from the in-silico $\mathrm{Hi}-\mathrm{C}$ maps in mESC returns a Spearman correlation between the bulk and the 60-cell map of $r_{s}=0.27$, close to the experimental value (Materials and Methods). Color scale indicates the percentiles of the maps. 

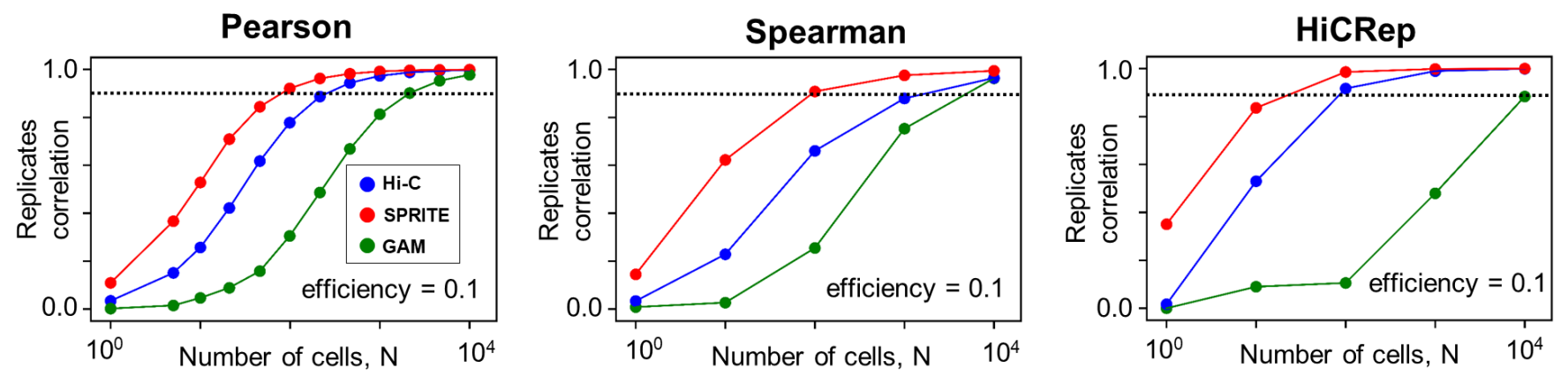

Supplementary Figure 5. Pearson, Spearman and HiCRep correlations between replicates in relation to the number of cells considered in the in-silico experiments.

The Pearson, Spearman and HiCRep correlations between replicate in-silico contact maps are shown for Hi-C, SPRITE and GAM at efficiency 0.1, in the case of the model of the Sox9 locus. Dashed lines in each plot indicates the considered 0.9 threshold value (see Figures 4c,d,e). 


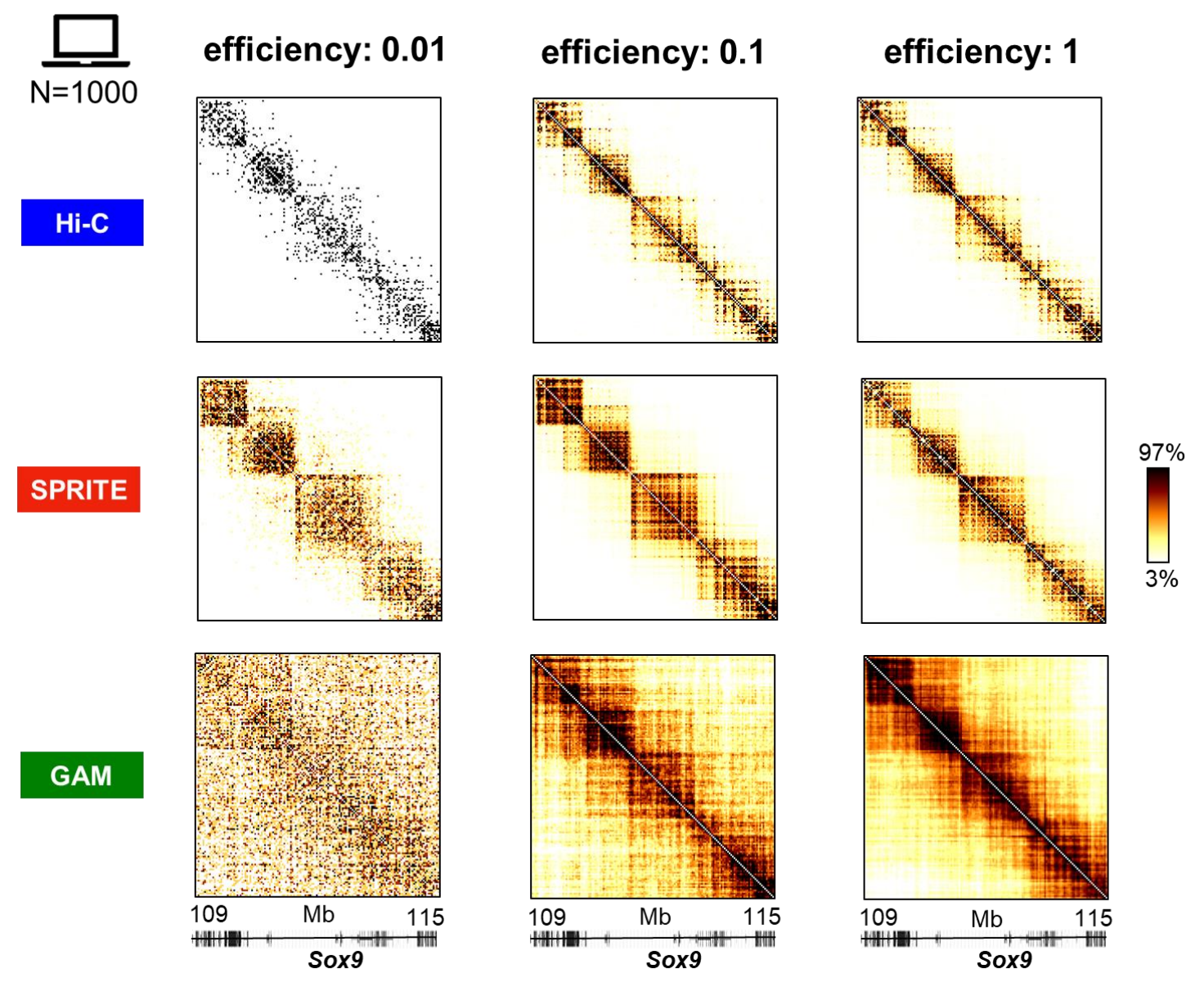

Supplementary Figure 6. Impact of the detection efficiency on in-silico contact maps.

Hi-C, SPRITE and GAM in-silico contact maps are shown for three different efficiencies $(0.01,0.1$ and 1.0) for a fixed $\mathrm{N}=1000$ in-silico cells, in the Sox9 locus case study. Low efficiencies can strongly disrupt the quality of the maps (see Figure $\mathbf{4 f}$ ) 

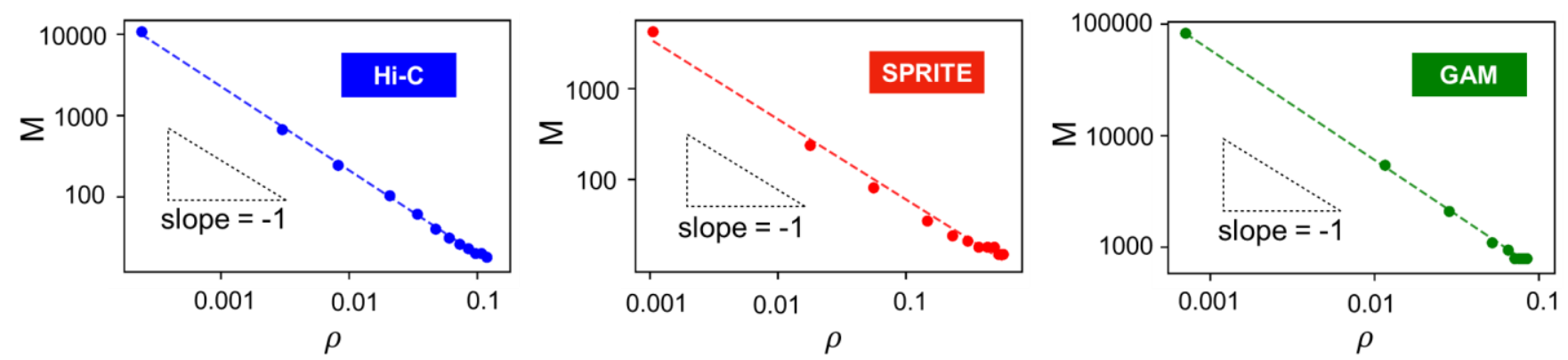

Supplementary Figure 7. The estimated value of $M$ is consistent with the Central-Limit-Theorem

In the Sox9 locus case study, the values of $M$ (see Main Text, Figure 4) at different efficiencies are plotted against $\rho$, the squared signal-to-noise ratio averaged over all the entries of a single-cell contact map (Materials and Methods). This is done for Hi-C (left), SPRITE (middle) and GAM (right). In all three plots (in log-log scale) the trend of $M$ vs $\rho$ is well fitted by a linear relationship with slope -1 (dashed lines) as expected by arguments based on the Central Limit Theorem (Materials and Methods). 
a)
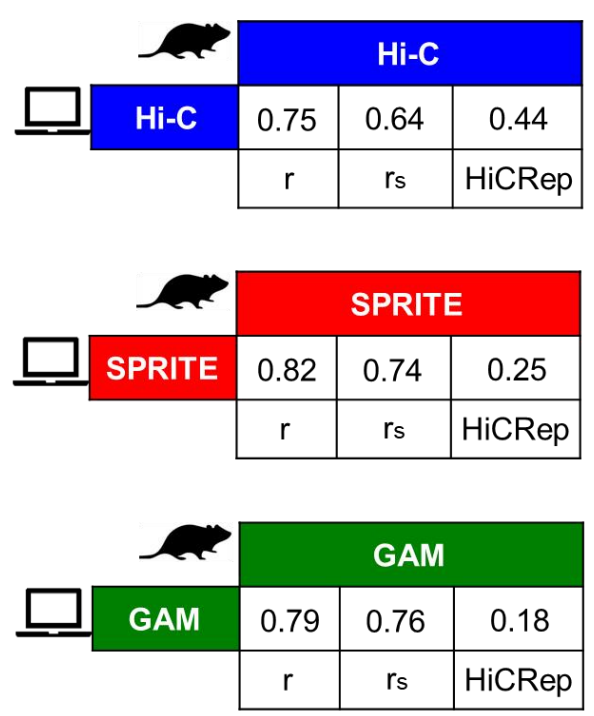

c)

\begin{tabular}{|c|c|c|c|}
\hline$\overline{\text { bulk }}$ & \multicolumn{3}{|c|}{ Distance } \\
\hline $\mathrm{Hi}-\mathrm{C}$ & -0.51 & -0.75 & -0.44 \\
\hline SPRITE & -0.59 & -0.83 & -0.45 \\
\hline GAM & -0.95 & -0.98 & -0.82 \\
\hline & $\mathrm{r}$ & rs & HiCRep \\
\hline
\end{tabular}

d)

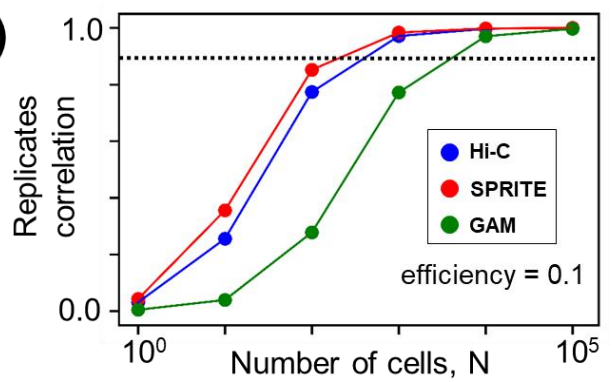

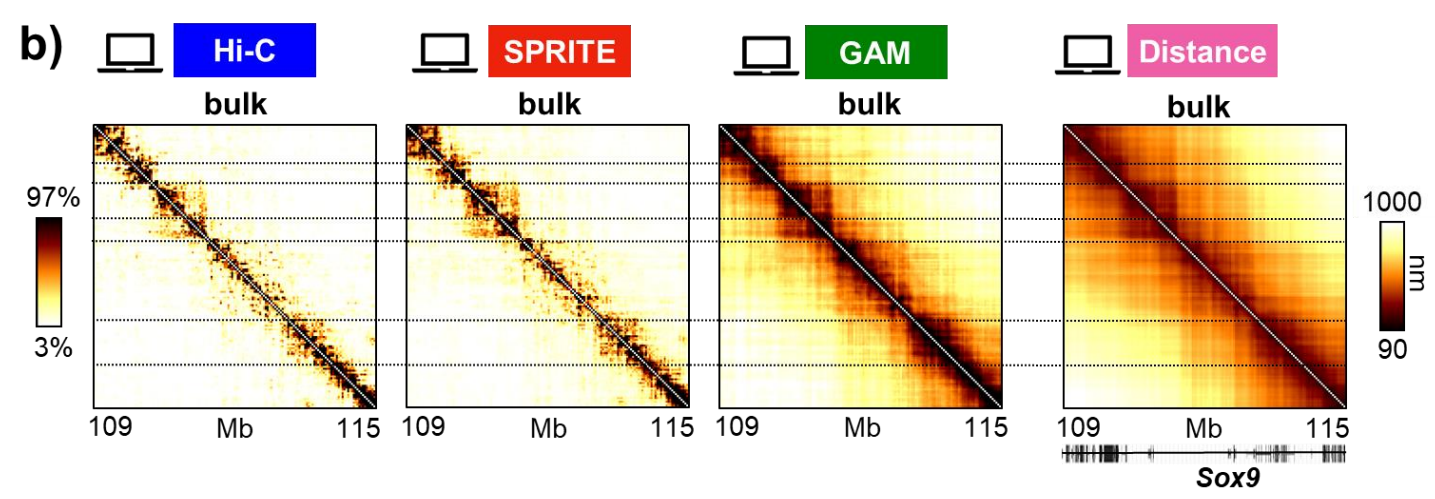

\section{Supplementary Figure 8. In-silico contact maps from 3D conformations derived from GAM data.}

a) We considered 3D structures for the mESC Sox9 locus derived from GAM data ${ }^{45}$ (Materials and Methods). The corresponding $\mathrm{Hi}-\mathrm{C}, \mathrm{SPRITE}$ and GAM in-silico contact maps are compared to the experimental data (the same used in Figure 1, see Materials and Methods) and their Pearson, Spearman and HiCRep correlations are reported.

b) The in-silico bulk contact maps are compatible with the average distance pattern obtained from the ensemble of GAM-derived 3D conformations. Horizontal lines are drawn to highlight the patterns detected across the contact and the distance maps. For the contact maps, color scale indicates the percentiles.

c) Pearson, Spearman and HiCRep correlations are reported between each bulk contact map and the average distance map.

d) Pearson correlation between replicates against the number of cells, for efficiency 0.1 . The dashed line is the threshold correlation value $r_{t}=0.9$. The values of $M$ in this case are analogous to the ones obtained for the Sox9, HoxD and Epha4 ensembles derived from Hi-C data, at the same 0.1 efficiency value (Figure 4d, Supplementary Figure 2c and Supplementary Figure 3d, respectively). 
a)

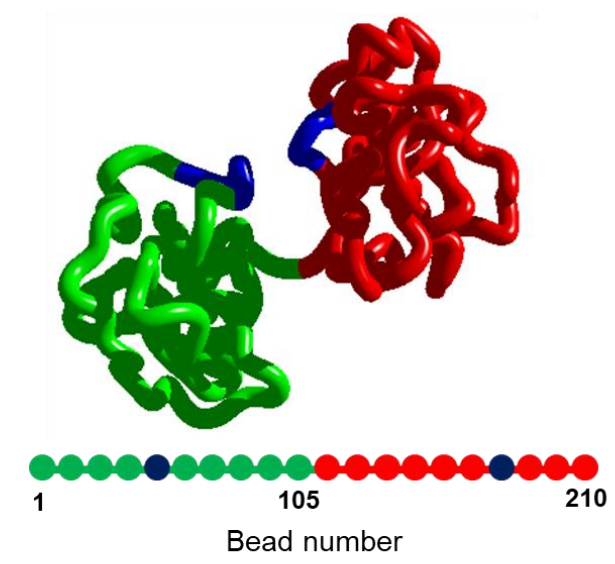

c)

\begin{tabular}{|c|c|c|c|}
\hline \multirow{2}{*}{$\frac{\square}{\text { bulk }}$} & \multicolumn{3}{|c|}{ Distance } \\
\hline & -0.64 & -0.89 & -0.72 \\
\hline SPRITE & -0.91 & -0.81 & -0.71 \\
\hline GAM & -0.91 & -0.89 & -0.72 \\
\hline & $r$ & rs & HiCRep \\
\hline
\end{tabular}

d)

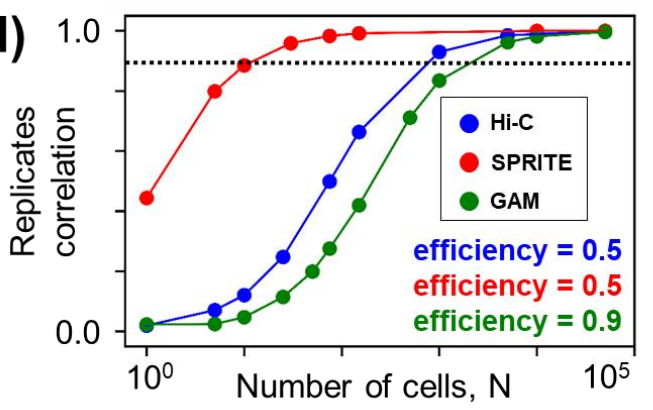

b)
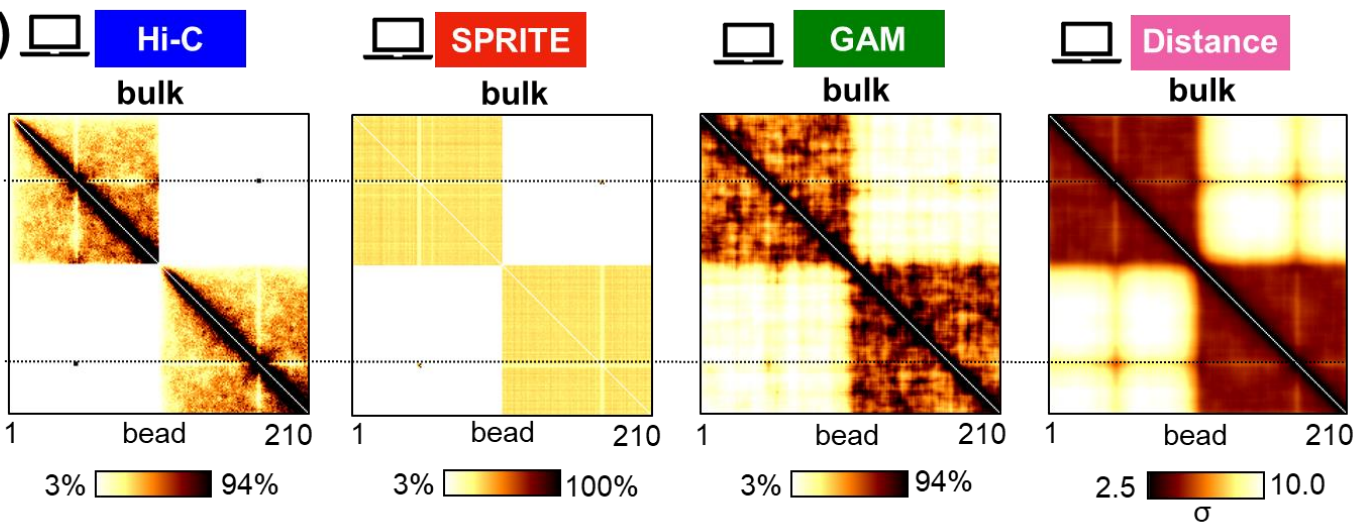

\section{Supplementary Figure 9. In-silico contact maps from 3D conformations of a toy polymer model.}

a) We considered a simple block copolymer model made of 210 beads where same colored regions attract each other (see Materials and Methods). The example of a 3D structure is shown.

b) In-silico Hi-C, SPRITE and GAM bulk contact maps all yield contact patterns compatible with the average distance pattern derived from our ensemble of conformations. The horizontal lines are a guide to the eye. Color scale for the contact maps indicates the percentiles. For the distance map, color scale is given in the units of $\sigma$, the diameter of a polymer bead (Materials and Methods).

c) The Pearson, Spearman and HiCRep correlations between each bulk contact and the average distance map are reported.

d) Replicate Pearson correlations are plotted v.s. the number of cells, $\mathrm{N}$, for: an efficiency equal to 0.5 for Hi-C and SPRITE, 0.9 for GAM. The values of $\mathrm{M}$ for the toy model are comparable to the ones obtained from the models of the Sox9, HoxD and Epha4 loci at similar efficiencies (Figures 4d,e; Supplementary Figure 2c, Supplementary Figure 3d, Supplementary Figure 8d). 

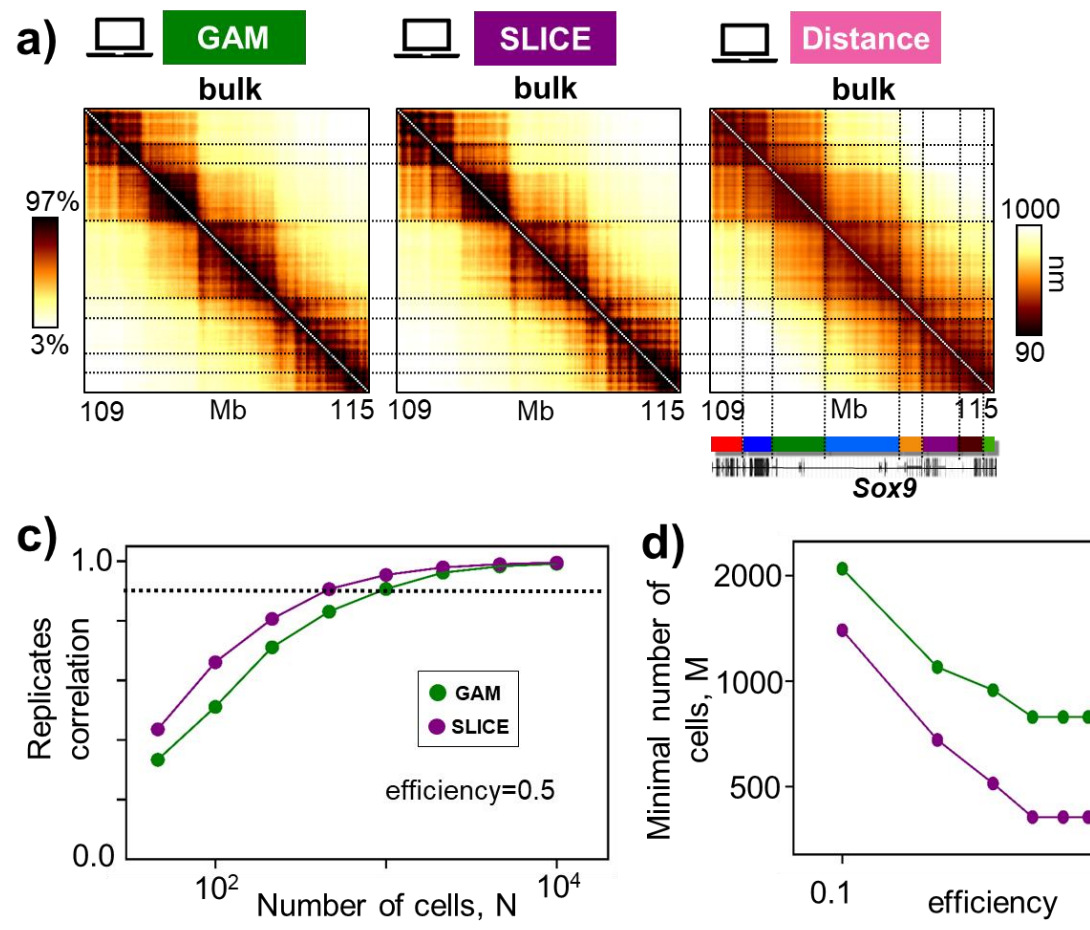
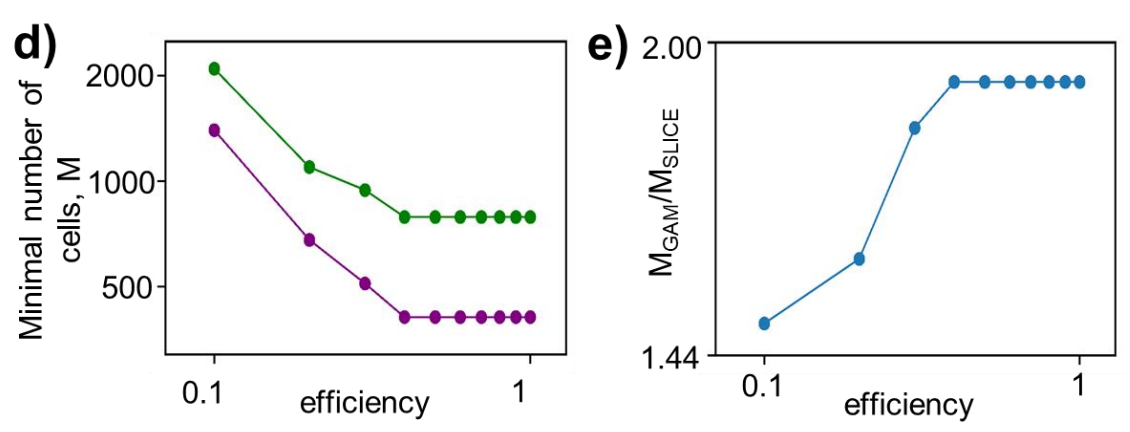

\section{Supplementary Figure 10. The SLICE analysis tool for GAM is faithful to the benchmark distance map.}

a) For the Sox9 locus case study, the in-silico bulk GAM map and the corresponding SLICE map (Main Text) return consistent interaction patterns. Color scale indicates the percentiles of the maps. In particular, the SLICE single-cell interaction probability map is also faithful to the average distance pattern. Horizontal lines highlight that GAM and SLICE both capture the domain structure of the distance map, corresponding to the TADs ${ }^{23}$ shown in the color bar at the bottom.

b) Pearson, Spearman and HiCRep correlations between SLICE and GAM bulk maps (top) and between SLICE and the average distance maps (bottom).

c) The Pearson correlation between replicate contact maps is shown as a function of the number of cells, N, for GAM and SLICE at efficiency 0.5 (Materials and Methods).

d) The minimal number of cells, M, to have reproducible replicates at different efficiencies for SLICE and GAM (as in Figure 4f).

e) The ratio between $M$ for GAM and for SLICE v.s. the efficiency. For efficiencies close to the experimental ones, say 0.5 or above ${ }^{20}$, the value of M for SLICE is approximately a factor two lower than for GAM. 
a)
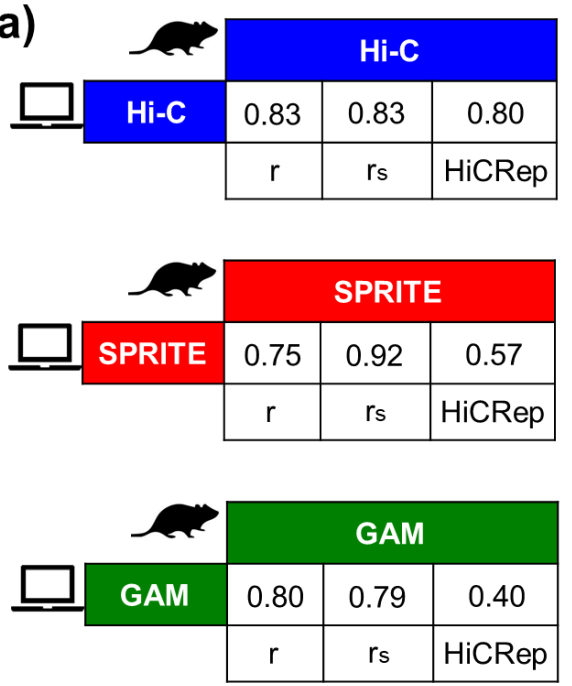

b)

\begin{tabular}{c|c|c|c|}
\multicolumn{1}{|c}{$\underline{\square}$ bulk } & \multicolumn{3}{|c|}{ Distance } \\
\cline { 2 - 4 } Hi-C & -0.54 & -0.89 & -0.73 \\
\hline SPRITE & -0.72 & -0.94 & -0.52 \\
\hline GAM & -0.94 & -0.99 & -0.93 \\
\hline & $r$ & rs & HiCRep \\
\cline { 2 - 4 } & & &
\end{tabular}

d)

\begin{tabular}{|c|c|c|c|}
\hline ngle cell & \multicolumn{3}{|c|}{ Distance } \\
\hline $\mathrm{Hi}-\mathrm{C}$ & -0.31 & -0.37 & -0.61 \\
\hline SPRITE & -0.37 & -0.46 & -0.57 \\
\hline GAM & -0.13 & -0.15 & -0.07 \\
\hline & $r$ & rs & HiCRep \\
\hline
\end{tabular}

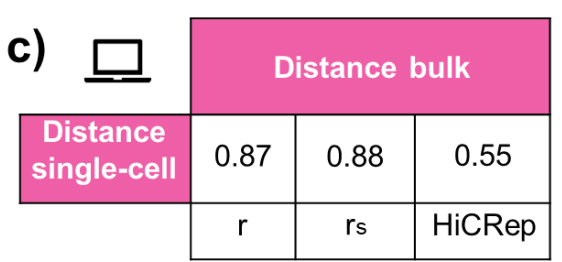

e)

\begin{tabular}{|c|c|c|c|}
\hline single cell & \multicolumn{3}{|c|}{ Distance } \\
\hline $\mathrm{Hi}-\mathrm{C}$ & -0.06 & -0.06 & -0.22 \\
\hline SPRITE & -0.13 & -0.16 & -0.36 \\
\hline GAM & -0.13 & -0.15 & -0.08 \\
\hline & $r$ & rs & HiCRep \\
\hline
\end{tabular}

Supplementary Table 1. Pearson, Spearman and HiCRep correlations for the Sox9 locus

a) Pearson ( $r$ ), Spearman $\left(r_{s}\right)$ and HiCRep (scc) correlations between bulk Hi-C, SPRITE and GAM (from 1122 slices) experimental maps and the corresponding in-silico maps (see Figure 1 and Materials and Methods), for the Sox9 locus.

b) Pearson, Spearman and HiCRep correlations between the average distance map and the in-silico bulk $\mathrm{Hi}-\mathrm{C}$, SPRITE and GAM contact maps (see Figure 2) for the Sox9 locus.

c) Pearson, Spearman and HiCRep mean correlations between in-silico single-cell distance maps and the average distance map for the Sox9 locus (see Figures $\mathbf{3 a , b}$ ).

d) Mean correlations between in-silico single-cell contact maps - at efficiency 1 - and the corresponding single-cell distance map (see Figures $\mathbf{3 c}, \mathbf{d}$ ).

e) Same as panel d), but here in-silico contact maps are generated with efficiencies similar to the experimental ones ( 0.05 for Hi-C and SPRITE and 0.5 for GAM; see Main Text and Materials and Methods). The reduction of efficiency worsens the similarity with the single-cell distance pattern. 